\title{
All Entrepreneurial Productivity Increases are Not Created Equal
}

\author{
by
}

Arup Bose, ${ }^{*}$ Debashis Pal, ${ }^{* *}$ and David E. M. Sappington***

\begin{abstract}
We examine the impact of productivity increases in a simple model of the entrepreneurial sector. Not surprisingly, many productivity increases generate gains for entrepreneurs and/or venture capitalists. However, some productivity increases generate Pareto losses. Furthermore, less capable entrepreneurs may benefit more from policies that increase the productivity of more capable entrepreneurs than from policies that increase their own productivity. Therefore, the design of policy to enhance welfare in the entrepreneurial sector can entail important subtleties.
\end{abstract}

February 2011

* Indian Statistical Institute, 203 B. T. Road, Kolkata 700108 India (bosearu@gmail.com).

** Department of Economics, University of Cincinnati, Cincinnati, Ohio 45221 USA (debashis.pal@uc.edu).

*** Department of Economics, University of Florida, PO Box 117140, Gainesville, FL 32611 USA (sapping@ufl.edu). 


\section{Introduction.}

The entrepreneurial sector is a large and growing component of many economies. ${ }^{1}$ Consequently, enhanced performance in the entrepreneurial sector can generate significant gains in the economy as a whole. An important task, therefore, is to identify activities that enhance the performance of the entrepreneurial sector. This research undertakes one element of this task by examining how several types of productivity increases affect performance in a simple, stylized model of the entrepreneurial sector. The productivity increases that we analyze can arise from many different sources, including reduced input prices, more efficient capital markets, and enhanced entrepreneurial skills generated by public or private training programs.

It would be natural to presume that any productivity increase in the entrepreneurial sector would increase the welfare of some or all sector participants. Indeed, governments often implement policies that are designed to improve the skills of entrepreneurs and salaried workers alike, and thereby increase welfare throughout the economy. ${ }^{2}$ Thus, it is not surprising that productivity increases of all types generate Pareto gains in our model when all parties have symmetric information about the potential returns from each entrepreneur's project. However, different conclusions can emerge when entrepreneurs are privately informed about the quality of their projects. In such settings, some productivity increases can even produce Pareto losses.

Some entrepreneurs in our model have access to high quality projects that are relatively likely to succeed. Other entrepreneurs only have access to low quality projects that are less likely to succeed. The difference in project success probabilities may reflect innate differences in project characteristics and/or differences in entrepreneurial skills. Entrepreneurs have no wealth, and so must borrow the funds required to implement their projects from a venture

\footnotetext{
${ }^{1}$ More than six million new businesses were created in the United States in 2009. This entrepreneurial activity "represents the highest level over the past decade and a half" (Fairlie, 2010, p. 2).

${ }^{2}$ For descriptions and assessments of selected policies, see Heckman (1998), Krueger and Rouse (1998), Heckman et al. (1999), Leuven and Oosterbeek (2004), and Abramovsky et al. (2011), for example.
} 
capitalist ("the lender"). Although each entrepreneur knows the quality of his project, the lender only observes an imperfect signal about project quality. The lender funds projects that generate a favorable signal and declines to fund projects that generate an unfavorable signal.

This representation of the entrepreneurial sector admits a straightforward analysis of the effects of different types of productivity increases. We consider increases in the revenue generated by successful projects, which might reflect enhanced product quality or improved marketing channels, for example. We also analyze the effects of reduced costs of funding and operating projects, which could stem from more efficient capital markets or lower costs of raw materials. In addition, we consider increases in the success rates of entrepreneurial projects, which could reflect the impact of training programs, for instance. In practice, some training programs are designed to improve basic skills, and so tend to be of primary benefit to entrepreneurs with relatively limited skills. ${ }^{3}$ Other training programs focus on developing more advanced capabilities and so tend to be of particular benefit to more highly skilled entrepreneurs. ${ }^{4}$

We find that different types of productivity increases can produce very different outcomes. To illustrate this conclusion, consider first the effect of an advanced training program that serves primarily to increase the probability that high quality projects succeed. This productivity increase induces the lender to increase the payment she promises to approved entrepreneurs whose projects ultimately succeed. The higher payment attracts more entrepreneurs and thereby increases the lender's profit. The higher payment also increases the welfare of all entrepreneurs, so Pareto gains arise.

\footnotetext{
${ }^{3}$ Government programs often focus on improving basic business skills. Abramovsky et al. (2011, pp. 154-5) describe the "Train to Gain" program in the UK which provides free training to individuals "who lack basic literacy, numeracy, or language skills." Also see Heckman et al. (1999).

${ }^{4}$ To illustrate, the Entrepreneurship \& Innovation Program at Northwestern University "is devoted to developing the next generation of high-growth entrepreneurs by giving students the theoretical and practical tools, networking opportunities and hands-on experience necessary to maximize their chances for success" (http://www.kellogg.northwestern.edu/ levyinstitute/program/index.htm). Stanford University's Program in Innovation and Entrepreneurship is designed to be a challenging "academic program for individuals formulating, developing and commercializing ideas" (http://www.gsb.stanford.edu/prie).
} 
A different conclusion can emerge when the productivity increase arises from a more basic training program that serves primarily to increase the probability that low quality projects succeed. This increased success probability renders entrepreneurs with low quality projects more anxious to have their projects funded. To deter excessive applications for funding, the lender may reduce the payment she promises to an entrepreneur whose project succeeds. The reduced payment reduces the welfare of entrepreneurs. The increased proportion of entrepreneurs with low quality projects that apply for funding also reduces the lender's profit. Thus, productivity increases can produce Pareto losses.

Our finding that productivity gains can generate systematic losses in the presence of asymmetric information is an illustration of the general theory of the second best (Lipsey and Lancaster, 1956-7). In this regard, our analysis is related to studies that show how an agent can enhance his well-being by reducing his capability. For example, Gelman and Salop (1983) demonstrate how a producer can profit by limiting its ability to expand output. ${ }^{5}$ Gupta et al. (1994, 1995) explain how a supplier can benefit from choosing inefficient locations or transportation costs. Fletcher and Slutsky (2010) show how a political candidate can benefit from a less favorable reputation.

In many of these studies, an agent benefits as his ability declines because his diminished capability induces a less aggressive response from a direct rival (e.g., a competing producer or an opposing political candidate). In our model, enhanced productivity can harm an entrepreneur because of the ensuing reaction of the supplier of a complementary input (the lender) rather than a rival. ${ }^{6}$ Our analysis also differs from its predecessors because we document Pareto losses from productivity gains whereas many other studies show that a productivity reduction can enable one party to gain at the expense of another. ${ }^{7}$

\footnotetext{
${ }^{5}$ Joskow and Kahn's (2002) observation that an electricity supplier can benefit by withholding electricity from the market can be viewed as a variation on this theme.

${ }^{6}$ Anant et al. (1995) show that a producer may gain by adopting an inefficient production technology that induces the government to impose a less stringent ad valorem tax.

${ }^{7}$ Bose et al. (2011) analyze a moral hazard setting in which a principal and agent can both gain as the agent's production costs increase.
} 
Our analysis proceeds as follows. Section 2 describes the key elements of our model. Section 3 analyzes a benchmark setting in which all parties share the same information $e x$ ante about the quality of each entrepreneur's project. Section 4 presents our main findings, emphasizing the conditions under which productivity increases generate systematic losses. Section 5 provides concluding observations and discusses some extensions of the analysis. The proofs of all formal conclusions are presented in the Appendix.

\section{Elements of the Model.}

We consider a setting where, as is common in practice, entrepreneurs have limited wealth and so rely on a lender (e.g., a venture capitalist) to finance their projects. The lender in our model has imperfect information about the likely payoff from the project of any particular entrepreneur. The sole lender employs the available screening technology to determine whether to finance the projects of entrepreneurs who apply for funding.

For simplicity, we assume that each entrepreneur has either a high quality project or a low quality project. A high quality project has a relatively high probability of success $\left(p_{H} \in(0,1)\right)$. A low quality project has a relatively low probability of success $\left(p_{L} \in\left(0, p_{H}\right)\right)$. A project generates payoff $V>0$ when it succeeds and 0 when it fails. Each project requires a fixed investment of $I>0$. A high quality project generates positive net surplus, i.e., $p_{H} V>I$. A low quality project generates negative net surplus, i.e., $p_{L} V<I$.

Each risk neutral entrepreneur knows the quality of his project. The risk neutral lender does not share this knowledge. Initially, the lender knows only that the fraction $\phi_{H} \in(0,1)$ of entrepreneurs have high quality projects and the complementary fraction $\phi_{L}=1-\phi_{H}$ have low quality projects. The lender eventually observes an informative signal $\left(s \in\left\{p_{L}, p_{H}\right\}\right)$ about the quality (i.e., the success probability) of the project of each entrepreneur that applies for funding. The signal reveals the true project quality with probability $q \in\left(\frac{1}{2}, 1\right)$ and reports the incorrect project quality with probability $1-q \cdot{ }^{8} \quad q$ is sufficiently large that

${ }^{8} q$ is assumed to exceed $\frac{1}{2}$ to ensure the signal is truly informative about project quality, and not simply pure noise. $q$ is assumed to be less than 1 to avoid unrealistic settings in which the lender can ascertain perfectly the quality of each lender's project. 
the lender optimally funds projects for which she observes the favorable signal and declines to fund projects for which she observes the unfavorable signal. ${ }^{9}$

Each entrepreneur experiences a transactions cost when applying for funding. This cost could reflect the time required to develop a compelling project description and associated business plan, for example. Variation in total transactions costs among entrepreneurs is captured in standard Hotelling fashion. Entrepreneurs with low quality projects (" $L$ entrepreneurs") and entrepreneurs with high quality projects (" $H$ entrepreneurs") are both distributed uniformly on the line segment $[0,1]$. The lender is located at 0 . The total number of entrepreneurs is normalized to 1 . An $L$ entrepreneur located at point $x$ incurs transactions cost $t_{L} x$ in applying for funding. The corresponding cost of the $H$ entrepreneur is $t_{H} x$. We will denote by $x_{i} \in\left\{x_{L}, x_{H}\right\}$ the location of the $i \in\{L, H\}$ entrepreneur farthest from the lender that applies for funding.

Entrepreneurs decide whether to apply for funding after observing the share of the payoff from a successful project $(\beta)$ the lender offers to approved entrepreneurs. An entrepreneur whose approved project fails receives a payoff of $0 .{ }^{10}$ An entrepreneur will apply for funding if and only if his expected return from doing so exceeds his transactions costs. Thus, an $L$ entrepreneur located at $x$ will apply for funding if and only if $\beta p_{L} V[1-q] \geq t_{L} x$. An $H$ entrepreneur located at $x$ will apply for funding if and only if $\beta p_{H} V q \geq t_{H} x$.

We assume that the unit transactions cost for $H$ entrepreneurs $\left(t_{H}\right)$ is not too much higher than the corresponding cost for $L$ entrepreneurs $\left(t_{L}\right)$ relative to the incremental surplus generated by a high quality project and the proportion of high quality projects in the population. This assumption avoids the uninteresting outcome in which the lender sets $\beta=0$ and thereby avoids funding any projects. Formally, Assumption 1 is assumed to hold

\footnotetext{
${ }^{9}$ We abstract from any cost of producing this informative signal, and take the screening accuracy $(q)$ to be exogenous. As explained in more detail in the concluding section, our primary findings persist in settings where $q$ is endogenous and the lender must incur higher costs in order to discern project qualities with greater accuracy.

${ }^{10}$ Among all feasible payment structures, this payment structure creates the strongest differential incentive for $H$ entrepreneurs to apply for funding. Consequently, this payment structure is optimal for the profit maximizing lender.
} 
throughout the ensuing analysis:

$\underline{\text { Assumption } 1 .}{ }^{11} \phi_{H} p_{H} q^{2} t_{L}\left[p_{H} V-I\right]+\phi_{L} p_{L} t_{H}[1-q]^{2}\left[p_{L} V-I\right]>0$.

The timing in the model is as follows. First, each entrepreneur privately learns the quality of his project. Second, the lender announces the sharing rate for approved projects. ${ }^{12}$ Third, entrepreneurs decide whether to seek funding for their project from the lender. Fourth, the lender assesses the project quality of each entrepreneur that applies for funding. The lender funds the projects that produce a favorable signal and declines to fund projects that produce an unfavorable signal. ${ }^{13}$ Finally, the outcome of each funded project is observed publicly. When an entrepreneur's project succeeds, he receives $\beta V$ and the lender receives $[1-\beta] V$.

The lender's (expected) profit in this setting when he sets sharing rate $\beta$ is:

$$
\pi(\beta)=\phi_{L} x_{L}[1-q]\left[p_{L} V(1-\beta)-I\right]+\phi_{H} x_{H} q\left[p_{H} V(1-\beta)-I\right]
$$

The first term to the right of the equality in (2) reflects the profit the lender anticipates from $L$ entrepreneurs. This profit is the product of the number of $L$ entrepreneurs that apply for funding $\left(\phi_{L} x_{L}\right)$, the probability that a low quality project is funded $(1-q)$, and the difference between the lender's expected payoff from a low quality project $\left(p_{L} V[1-\beta]\right)$ and the cost of financing the project $(I)$. The last term in (2) reflects the corresponding profit the lender anticipates from $H$ entrepreneurs.

The ensuing analysis examines the effects of productivity increases on the lender's profit and on the welfare of entrepreneurs. The welfare of $L$ entrepreneurs, given sharing rate $\beta$, is:

$$
W_{L}(\beta)=\phi_{L} x_{L}[1-q] p_{L} V \beta-\phi_{L} t_{L} \int_{0}^{x_{L}} x d x .
$$

\footnotetext{
${ }^{11}$ As demonstrated in the proof of Lemma 3, assumption 1 ensures that the lender's profit is strictly increasing in $\beta$ at $\beta=0$ for all $q \in\left(\frac{1}{2}, 1\right)$.

${ }^{12}$ We assume that the lender can make a binding commitment to deliver the promised sharing rate. In doing so, we abstract from the possibility that the lender might try to expropriate entrepreneurs by reducing $\beta$ after they apply for funding. Reputation concerns can promote such commitment, for example.

${ }^{13}$ Entrepreneurs whose request for funding is denied, like entrepreneurs who do not seek funding, earn 0 (in the wage sector of the economy, for example).
} 
The first term to the right of the equality in (3) is the product of the number of entrepreneurs whose projects are funded $\left(\phi_{L} x_{L}[1-q]\right)$ and an $L$ entrepreneur's expected payoff from a funded project $\left(p_{L} V \beta\right)$. The second term to the right of the equality in (3) reflects the aggregate transactions costs of $L$ entrepreneurs that apply for funding.

The corresponding welfare of $H$ entrepreneurs, given sharing rate $\beta$, is:

$$
W_{H}(\beta)=\phi_{H} x_{H} q p_{H} V \beta-\phi_{H} t_{H} \int_{0}^{x_{H}} x d x .
$$

We will consider two types of productivity increases: increases in the expected payoff of a project and reductions in the cost of project implementation. The expected payoff of a project increases when the payoff from success $(V)$ increases and/or the probability of success $\left(p_{L}\right.$ or $\left.p_{H}\right)$ increases. The cost of project implementation declines when the financing cost (I) declines and/or transactions costs $\left(t_{L}\right.$ or $\left.t_{H}\right)$ decline. As the analysis in Section 4 reveals, these different types of productivity increases can produce qualitatively different effects.

\section{Benchmark Setting with Symmetric Ex Ante Information.}

Before analyzing the effects of productivity increases in the setting described in Section 2, we briefly consider these effects in the following symmetric information setting. In this benchmark setting, each entrepreneur and the lender initially share the same imperfect knowledge of the quality of the entrepreneur's project. It is common knowledge that an entrepreneur has a high quality project with probability $\phi_{H}$ and a low quality project with probability $\phi_{L}$. Each entrepreneur decides whether to apply for funding and pursue his project before learning the quality of his project. All parties also know that an informative signal about the quality of each entrepreneur's project will be produced if the entrepreneur applies for funding. A high quality project generates a favorable signal (and so is funded) with probability $q \in\left(\frac{1}{2}, 1\right)$ and an unfavorable signal (and so is not funded) with probability $1-q$. A low quality project generates a favorable signal (and so is funded) with probability $1-q$ and an unfavorable signal (and so is not funded) with probability $q$. For simplicity, 
each entrepreneur is assumed to face the same unit transactions cost in seeking funding, i.e., $t_{L}=t_{H}=t .^{14}$

Lemma 1 explains how the various productivity increases under consideration affect the profit maximizing sharing rate.

Lemma 1. The lender increases the sharing rate $(\beta)$ in the symmetric information setting as $V, p_{L}, p_{H}$, and/or $\phi_{H}$ increase, and as $I$ decreases.

The conclusions reported in Lemma 1 reflect standard considerations. The expected net surplus from each entrepreneur's project increases as $V, p_{L}, p_{H}$, and/or $\phi_{H}$ increase and as $I$ decreases. Consequently, the lender increases the sharing rate in order to induce more entrepreneurs to apply for funding. ${ }^{15}$

The lender's optimal response to productivity increases in this setting produces Pareto gains, as Observation 1 reports.

Observation 1. The lender's expected profit and the entrepreneurs' expected welfare both increase in the symmetric information setting as: (i) $V, p_{L}, p_{H}$, and/or $\phi_{H}$ increase; and (ii) $I$ and/or $t$ decline.

The lender benefits from the increased potential profit generated by the various productivity increases considered in Observation 1. The entrepreneurs benefit from the increased sharing rate and, when relevant, their reduced transactions costs or the increased expected payoff from their projects.

The conclusions reported in Observation 1 are not surprising. It is natural to expect productivity increases to generate gains that are shared by the participants in the entrepreneurial sector of an economy. What may be more surprising is that productivity gains can

\footnotetext{
${ }^{14}$ We also assume that $\phi_{H} q\left[p_{H} V-I\right]+\phi_{L}[1-q]\left[p_{L} V-I\right]>0$ in the symmetric information setting to ensure the lender optimally sets $\beta>0$.

${ }^{15}$ As the proof of Lemma 1 demonstrates, the profit maximizing sharing rate does not vary with $t$ in the symmetric information setting. As $t$ declines, a given increase in $\beta$ induces more entrepreneurs to apply for funding, which encourages the lender to increase $\beta$. However, a reduction in $t$ renders entrepreneurs more willing to apply for funding even when $\beta$ is low, which leads the lender to reduce $\beta$. These two effects are offsetting in the symmetric information setting.
} 
produce Pareto losses in arguably more realistic settings where entrepreneurs initially have private information about the quality of their projects. ${ }^{16}$

\section{Main Findings.}

We now return to the setting of primary interest in which each entrepreneur is privately informed about the quality of his project. We begin by identifying the locations of the $L$ entrepreneur $\left(x_{L}\right)$ and the $H$ entrepreneur $\left(x_{H}\right)$ farthest from the lender that apply for funding, given sharing rate $\beta$.

Lemma 2. $x_{L}=\beta p_{L} V\left[\frac{1-q}{t_{L}}\right]$ and $x_{H}=\beta p_{H} V\left[\frac{q}{t_{H}}\right]$.

Lemma 2 reflects the obvious conclusion that more entrepreneurs will apply for funding as the expected payoff from their project $\left(p_{i} V\right)$ increases, as the sharing rate $(\beta)$ increases, as the probability that their project will be funded increases, and as their transactions costs $\left(t_{i}\right)$ decline.

Lemma 2 helps to characterize the equilibrium (profit maximizing) sharing rate $\left(\beta^{*}\right)$, the lender's equilibrium profit $\left(\pi^{*}=\pi\left(\beta^{*}\right)\right)$, the equilibrium welfare of $L$ entrepreneurs $\left(W_{L}^{*}=\right.$ $\left.W_{L}\left(\beta^{*}\right)\right)$, the equilibrium welfare of $H$ entrepreneurs $\left(W_{H}^{*}=W_{H}\left(\beta^{*}\right)\right)$, and equilibrium total welfare $\left(W^{*}=\pi^{*}+W_{L}^{*}+W_{H}^{*}\right)$. These measures are specified in Lemmas $3-5$.

Lemma 3. The equilibrium sharing rate is:

$$
\beta^{*}=\frac{\phi_{L} p_{L}[1-q]^{2}\left[p_{L} V-I\right] t_{H}+\phi_{H} p_{H} q^{2}\left[p_{H} V-I\right] t_{L}}{2 V\left[\phi_{L} p_{L}^{2}(1-q)^{2} t_{H}+\phi_{H} p_{H}^{2} q^{2} t_{L}\right]} .
$$

Lemma 4. The lender's equilibrium profit is:

$$
\pi^{*}=\frac{\left\{\phi_{L} p_{L}[1-q]^{2} t_{H}\left[p_{L} V-I\right]+\phi_{H} p_{H} q^{2} t_{L}\left[p_{H} V-I\right]\right\}^{2}}{4 t_{L} t_{H}\left[\phi_{L} p_{L}^{2} t_{H}(1-q)^{2}+\phi_{H} p_{H}^{2} t_{L} q^{2}\right]}
$$

\footnotetext{
${ }^{16}$ Even this finding is not entirely surprising since, as noted in the Introduction, it is an illustration of the general theory of the second best (Lipsey and Lancaster, 1956-7).
} 
Lemma 5. The equilibrium welfare of $L$ entrepreneurs, the equilibrium welfare of $H$ entrepreneurs, and equilibrium total welfare are, respectively:

$$
\begin{aligned}
W_{L}^{*} & =\frac{\phi_{L} p_{L}^{2}[1-q]^{2}\left\{\phi_{L} p_{L}[1-q]^{2} t_{H}\left[p_{L} V-I\right]+\phi_{H} p_{H} q^{2} t_{L}\left[p_{H} V-I\right]\right\}^{2}}{8 t_{L}\left[\phi_{L} p_{L}^{2}(1-q)^{2} t_{H}+\phi_{H} p_{H}^{2} q^{2} t_{L}\right]^{2}} ; \\
W_{H}^{*} & =\frac{\phi_{H} p_{H}^{2} q^{2}\left\{\phi_{L} p_{L}[1-q]^{2} t_{H}\left[p_{L} V-I\right]+\phi_{H} p_{H} q^{2} t_{L}\left[p_{H} V-I\right]\right\}^{2}}{8 t_{H}\left[\phi_{L} p_{L}^{2}(1-q)^{2} t_{H}+\phi_{H} p_{H}^{2} q^{2} t_{L}\right]^{2}} ; \text { and } \\
W^{*} & =\frac{3\left\{\phi_{L} p_{L}[1-q]^{2} t_{H}\left[p_{L} V-I\right]+\phi_{H} p_{H} q^{2} t_{L}\left[p_{H} V-I\right]\right\}^{2}}{8 t_{L} t_{H}\left[\phi_{L} p_{L}^{2}(1-q)^{2} t_{H}+\phi_{H} p_{H}^{2} q^{2} t_{L}\right]} .
\end{aligned}
$$

Now consider how the lender adjusts the equilibrium sharing rate in response to various productivity increases.

Lemma 6. The equilibrium sharing rate $\left(\beta^{*}\right)$ increases as: (i) $V, p_{H}, \phi_{H}$, and/or $t_{L}$ increase; and (ii) I and/or $t_{H}$ decrease.

The conclusions in Lemma 6 reflect the following five considerations. First, as $V$ increases and/or $I$ declines, each project generates more surplus. Consequently, the lender increases the sharing rate $(\beta)$ in order to induce more entrepreneurs to apply for funding. Second, as $p_{H}$ increases, a given increase in $\beta$ attracts more $H$ entrepreneurs. ${ }^{17}$ Therefore, the lender increases $\beta$ as $p_{H}$ increases to attract more high quality projects, which offer greater potential profit as $p_{H}$ increases.

Third, as the proportion of $H$ entrepreneurs in the population $\left(\phi_{H}\right)$ increases, a given increase in $\beta$ attracts a higher proportion of high quality projects. Consequently, the lender's profit increases more rapidly as $\beta$ increases, which leads the lender to set a higher sharing rate. Fourth, when $L$ entrepreneurs incur higher transactions costs (i.e., as $t_{L}$ increases), they find it more burdensome to apply for funding. Consequently, the lender can increase $\beta$ to attract more high quality projects without fear of attracting too many low quality ${ }^{17}$ Formally, from Lemma $2, \frac{\partial^{2} x_{H}}{\partial p_{H} \partial \beta}=\frac{V q}{t_{H}}>0$. 
projects. Fifth, when $H$ entrepreneurs incur smaller transactions costs (so $t_{H}$ declines), an increase in $\beta$ becomes more effective at inducing them to apply for funding. Consequently, the lender increases $\beta$ to attract the profitable high quality projects.

The impact of an increase in $p_{L}$ on the profit-maximizing sharing rate is less straightforward, as Lemma 7 suggests.

Lemma 7. As the success probability of a low quality project $\left(p_{L}\right)$ increases, the equilibrium sharing rate $\left(\beta^{*}\right)$ declines if $p_{L} \leq \widetilde{p}_{L}$ whereas $\beta^{*}$ increases if $p_{L}>\widetilde{p}_{L}$, where $\frac{\widetilde{p}_{L}}{p_{H}}=\delta_{1}=$ $\frac{\sqrt{1+\delta_{0}}-1}{\delta_{0}}$ and $\delta_{0}=\frac{\phi_{L}[1-q]^{2} t_{H}}{\phi_{H} q^{2} t_{L}}$.

An increase in $p_{L}$ has two countervailing effects. First, it renders the decision of $L$ entrepreneurs to seek funding more sensitive to the prevailing sharing rate, $\beta .{ }^{18}$ This effect leads the lender to reduce $\beta$ in order to discourage $L$ entrepreneurs from applying for funding. Second, the lender incurs a smaller loss on each low quality project she finances. This effect leads the lender to increase $\beta$ (in order to attract more $H$ entrepreneurs). The second effect is of greater importance when $p_{L}$ is relatively large, and so the lender finances relatively many low quality projects. ${ }^{19}$

The conclusions in Lemmas $6-7$ help to explain the effects of productivity increases on equilibrium profit and welfare. Proposition 1 identifies productivity increases that generate Pareto gains.

Proposition 1. The lender's profit $\left(\pi^{*}\right)$ and the welfare of both $L$ and $H$ entrepreneurs ( $W_{L}^{*}$ and $W_{H}^{*}$ ) increase as: (i) $V$ and/or $p_{H}$ increase; and (ii) $I$ and/or $t_{H}$ decline.

The Pareto gains identified in Proposition 1 reflect the following four considerations. First, an increase in $V$ increases the lender's profit by increasing the expected payoff from each entrepreneur's project. The increased profit induces the lender to increase $\beta$ in order to

\footnotetext{
${ }^{18}$ Formally, from Lemma $2, \frac{\partial^{2} x_{L}}{\partial \beta \partial p_{L}}=\frac{V[1-q]}{t_{L}}>0$.

${ }^{19}$ Notice from Lemma 2 that $\frac{x_{L}}{x_{H}}=\frac{p_{L}[1-q] t_{H}}{p_{H} q t_{L}}$, which increases as $p_{L}$ increases.
} 
attract more entrepreneurs, which increases their welfare. Second, a reduction in $I$ increases the lender's expected profit by reducing her investment costs. In response to the increased profitability of each project, the lender increases $\beta$ in order to attract more entrepreneurs, which increases their welfare.

Third, as $t_{H}$ declines, a given increase in $\beta$ induces more $H$ entrepreneurs to apply for funding. ${ }^{20}$ Consequently, the lender increases $\beta$ in order to attract more high quality projects. The lender's profit increases as she finances a higher proportion of profitable projects, and all entrepreneurs benefit from the higher sharing rate. Fourth, an increase in $p_{H}$ increases the profit the lender secures from each high quality project she finances. An increase in $p_{H}$ also increases the sensitivity of the decision of $H$ entrepreneurs to apply for funding to the prevailing sharing rate. ${ }^{21}$ Consequently, the lender increases $\beta$ in order to attract more $H$ entrepreneurs, which increases the welfare of all entrepreneurs.

Proposition 1 demonstrates that several forms of productivity gains generate Pareto improvements. Proposition 2 considers the impact of one additional form of productivity gain - an increase in the proportion of high quality projects in the population $\left(\phi_{H}\right) \cdot{ }^{22}$ The proposition refers to the following assumption.

Assumption 2. $\quad \frac{\left[1-\phi_{H}\right]\left[2-\phi_{H}\right]}{\left(\phi_{H}\right)^{2}}>\frac{p_{H}^{2} q^{2} t_{L}}{p_{L}^{2}[1-q]^{2} t_{H}}$.

Proposition 2. The lender's profit $\left(\pi^{*}\right)$ and the welfare of $H$ entrepreneurs $\left(W_{H}^{*}\right)$ increase as the proportion of $H$ entrepreneurs $\left(\phi_{H}\right)$ increases. The welfare of $L$ entrepreneurs $\left(W_{L}^{*}\right)$ also increases if Assumptions 1 and 2 hold.

An increase in the proportion of high quality projects increases the lender's profit directly. It also induces the lender to increase $\beta$ in order to attract more $H$ entrepreneurs. The increase

${ }^{20}$ Formally, from Lemma 2, $-\frac{\partial^{2} x_{H}}{\partial t_{H} \partial \beta}=\frac{p_{H}}{\left(t_{H}\right)^{2}}>0$.

${ }^{21}$ Formally, from Lemma 2, $\frac{\partial^{2} x_{H}}{\partial p_{H} \partial \beta}=\frac{q V}{t_{H}}>0$.

${ }^{22} \mathrm{An}$ increase in $\phi_{H}$ might arise from a training program that transforms some low-skilled entrepreneurs into high-skilled entrepreneurs, for example. 
in $\beta$ increases the welfare of $H$ entrepreneurs. It can also increase the aggregate welfare of $L$ entrepreneurs under many plausible settings, including those in which Assumption 2 holds. Assumption 2 will be satisfied, for example, when the transactions costs of $L$ entrepreneurs are relatively small. ${ }^{23}$ When $t_{L}$ is relatively small, the increase in the sharing rate induces many $L$ entrepreneurs to apply for funding. This source of increased participation for $L$ entrepreneurs can outweigh the reduced participation that arises from the decline in the proportion of $L$ entrepreneurs in the population. ${ }^{24}$

The discussion to this point has focused on productivity increases that generate Pareto gains. In contrast, Proposition 3 identifies a productivity increase that can generate Pareto losses. The proposition refers to the following assumption.

Assumption 3. $\quad \frac{\phi_{L}}{\phi_{H}}>\frac{p_{H}^{2} q^{2} t_{L}}{p_{L}^{2}[1-q]^{2} t_{H}}$.

Proposition 3. The lender's profit $\left(\pi^{*}\right)$, the welfare of $H$ entrepreneurs $\left(W_{H}^{*}\right)$, and total welfare $\left(W^{*}\right)$ all decline as the transactions costs of $L$ entrepreneurs $\left(t_{L}\right)$ decline. The welfare of $L$ entrepreneurs $\left(W_{L}^{*}\right)$ also declines as $t_{L}$ declines if Assumptions 1 and 3 hold.

When the transactions costs that $L$ entrepreneurs experience decline, the lender reduces $\beta$ to avoid attracting too many low quality projects. This reduction in $\beta$ reduces the welfare of the $H$ entrepreneurs. In contrast, the reduction in their transactions costs can increase the welfare of $L$ entrepreneurs. However, their welfare can decline in plausible settings, including those in which $L$ entrepreneurs experience substantially smaller transactions costs than $H$ entrepreneurs. When $t_{L}$ is small, an $L$ entrepreneur's decision about whether to seek financing is relatively sensitive to changes in $\beta$. Consequently, the reduction in $\beta$ induces many $L$ entrepreneurs not to apply for funding, which reduces their welfare. ${ }^{25}$

\footnotetext{
${ }^{23} \mathrm{~A}$ small value of $t_{L} / t_{H}$ could arise, for example, when $L$ entrepreneurs have a relatively small opportunity cost of applying for funding.

${ }^{24} W_{L}^{*}$ can increase as $\phi_{H}$ increases even when $t_{L} / t_{H}$ is not small. (Equation (31) in the Appendix provides a less stringent sufficient condition for $\frac{\partial W_{L}^{*}}{\partial \phi_{H}}>0$.) For example, it is readily verified that $W_{L}^{*}$ increase as $\phi_{H}$ increases between 0.40 and 0.45 when $V=40, I=20, p_{H}=0.6, p_{L}=0.25, q=0.7$, and $t_{L}=t_{H}=4$.

${ }^{25} W_{L}^{*}$ can decline as $t_{L}$ declines even when $t_{L} / t_{H}$ is not small. (As the proof of Proposition 3 reveals,
} 
Proposition 3 provides an example of a particular form of productivity increase that can produce Pareto losses. Proposition 4 provides an additional example of a productivity increase that can produce systematic gains or losses, depending upon the environment in which the productivity increase arises.

Proposition 4. The lender's profit $\left(\pi^{*}\right)$, the welfare of $H$ entrepreneurs $\left(W_{H}^{*}\right)$, and aggregate welfare $\left(W^{*}\right)$ all decline as the success probability of a low quality project $\left(p_{L}\right)$ increases when $p_{L}$ is sufficiently small. The welfare of $L$ entrepreneurs $\left(W_{L}^{*}\right)$ also can decline ${ }^{26}$ and so an increase in $p_{L}$ can generate Pareto losses. In contrast, $\pi^{*}, W_{H}^{*}$, and $W_{L}^{*}$ all increase as $p_{L}$ increases when $p_{L}$ and $q$ are sufficiently large.

When $p_{L}$ is small, relatively few $L$ entrepreneurs apply for funding. (Recall Lemma 2.) Because she does not fund the projects of many $L$ entrepreneurs, the lender does not secure much of an increase in profit as the success probability of low quality projects increases. Furthermore, the increase in $p_{L}$ renders the number of $L$ entrepreneurs who apply for funding more sensitive to $\beta$. To discourage the $L$ entrepreneurs from applying for funding, the lender reduces the sharing rate, $\beta$. (Recall Lemma 7.) The reduction in $\beta^{*}$ reduces the welfare of the $H$ entrepreneurs $\left(W_{H}^{*}\right)$. The increased relative participation of $L$ entrepreneurs reduces the lender's profit $\left(\pi^{*}\right)$. The reduction in $W_{H}^{*}$ and $\pi^{*}$ cause aggregate welfare $\left(W^{*}\right)$ to decline, even when the welfare of $L$ entrepreneurs $\left(W_{L}^{*}\right)$ increases. The reduction in $\beta^{*}$ induced by an increase in $p_{L}$ can be so pronounced as to diminish the welfare of $L$ entrepreneurs.

This outcome is illustrated in Table 1 in the setting where a successful project generates $V=40$, each project requires investment $I=20$, sixty percent of entrepreneurs have low quality projects (so $\phi_{L}=0.6$ and $\phi_{H}=0.4$ ), $L$ and $H$ entrepreneurs experience the same

\footnotetext{
$\frac{\partial W_{L}^{*}}{\partial \phi_{H}}>0$ when the function $g(\cdot)$ defined in equation (33) in the Appendix is positive.) For example, it is readily verified that $W_{L}^{*}$ decreases as $t_{L}$ declines between 6.0 and 4.0 when $V=40, I=20, p_{H}=0.6$, $p_{L}=0.25, q=0.7, \phi_{L}=.7$, and $t_{H}=4$.

${ }^{26}$ As the proof of Proposition 4 reveals, $\frac{\partial W_{L}^{*}}{\partial p_{L}}<0$ if $\left[A p_{L}^{2}+p_{H}\right]^{2} V<\left[p_{H}\left(1+2 A p_{L}\right)-A p_{L}^{2}\right] I$, where $A=\frac{\phi_{L}[1-q]^{2} t_{H}}{\phi_{H} p_{H} q^{2} t_{L}}$.
} 
transactions costs (so $t_{L}=t_{H}=4$ ), high quality projects succeed with probability 0.6, and the screening accuracy is $q=0.55$. The table presents the changes in the equilibrium sharing rate $\left(\beta^{*}\right)$, the lender's profit $\left(\pi^{*}\right)$, the welfare of entrepreneurs $\left(W_{L}^{*}\right.$ and $\left.W_{H}^{*}\right)$, and total welfare $\left(W^{*}\right)$ that arise as $p_{L}$ increases from 0.07 to $0.11 .{ }^{27}$ Here and more generally, Pareto losses from an increase in $p_{L}$ are most likely when $p_{L}$ is moderately small. ${ }^{28}$ Table 1 reveals that these losses can be substantial. To illustrate, as $p_{L}$ increases by $10 \%$, from .10 to .11 , aggregate welfare declines by more than $27 \%$. This decline reflects a reduction of (approximately) 27\% in the lender's profit, $28 \%$ in the welfare of $H$ entrepreneurs, and $13 \%$ in the welfare of $L$ entrepreneurs.

\begin{tabular}{|cccccc|}
\hline$p_{L}$ & $\beta^{*}$ & $\pi^{*}$ & $W_{L}^{*}$ & $W_{H}^{*}$ & $W^{*}$ \\
\hline 0.07 & 40.7974 & 29.3974 & 0.1982 & 14.5005 & 44.0961 \\
0.08 & 35.8341 & 22.7733 & 0.1997 & 11.187 & 34.1600 \\
0.09 & 31.1674 & 17.3082 & 0.1912 & 8.4629 & 25.9623 \\
0.10 & 26.8007 & 12.8643 & 0.1745 & 6.2576 & 19.2965 \\
0.11 & 22.7364 & 9.3113 & 0.1512 & 4.5036 & 13.9669 \\
\hline
\end{tabular}

Table 1. Pareto Losses from an Increase in $p_{L}$.

In contrast, when $p_{L}$ is large, relatively many $L$ entrepreneurs apply for and receive funding if the lender's screening accuracy $(q)$ is sufficiently limited. In this case, an increase in $p_{L}$ reduces the loss the lender incurs on each low quality project she funds. Consequently, the lender becomes less intent on avoiding low quality projects, and so increases $\beta^{*}$ in order to attract more high quality projects. The increase in $\beta^{*}$ increases $W_{L}^{*}$ and $W_{H}^{*}$. Therefore,

${ }^{27}$ This is approximately the range of $p_{L}$ realizations in which the lender secures more profit by financing only projects that generate a positive signal than by financing the projects of all entrepreneurs that apply for funding. For expositional ease, the entries in all columns but the first in Table 1 represent 1,000 times the actual relevant value.

${ }^{28}$ When $p_{L}$ is close to 0 , the lender funds the projects of very few $L$ entrepreneurs and so does not reduce $\beta^{*}$ much, if at all, as $p_{L}$ increases. Consequently, an increase in $p_{L}$ serves primarily to increase the welfare of $L$ entrepreneurs as more of them apply for funding. Formally, as shown in equation (22) in the proof of Lemma $5, W_{L}^{*}=\frac{\phi_{L}[1-q]^{2}\left(\beta p_{L} V\right)^{2}}{2 t_{L}}$. Consequently, $\frac{d W_{L}^{*}}{d p_{L}} \stackrel{s}{=} \beta^{*}+p_{L} \frac{d \beta^{*}}{d p_{L}}>0$ for $p_{L}$ sufficiently close to 0 . 
Pareto gains can arise as $p_{L}$ increases in this setting with asymmetric information, just as they arise in the benchmark symmetric information setting.

When the lender's ability to identify project quality $(q)$ is sufficiently pronounced, the lender can simply decline to finance what he considers to be low quality projects rather than discourage $L$ entrepreneurs from applying for funding by reducing $\beta$. Consequently, the lender does not reduce $\beta$ much, if at all, as $p_{L}$ increases when $q$ is relatively large. Therefore, $L$ entrepreneurs benefit from an increase in $p_{L}$ when the lender has sufficient ability to distinguish between low quality projects and high quality projects. As Corollary 1 indicates, this ability need not be particularly pronounced.

Corollary 1. The welfare of $L$ entrepreneurs $\left(W_{L}^{*}\right)$ increases as the success probability of a low quality project $\left(p_{L}\right)$ increases if $q \geq \frac{\sqrt{2}}{1+\sqrt{2}} \approx 0.586$.

Before concluding, we present and illustrate the following implication of Propositions 1 and 4 .

Corollary 2. An increase in $p_{H}$ can increase the welfare of $L$ entrepreneurs by more than a commensurate increase in $p_{L}$.

Corollary 2 implies that $L$ entrepreneurs can benefit more from a training program (or technological change) that enhances the performance of $H$ entrepreneurs than from a program that improves their own performance. This more pronounced gain for $L$ entrepreneurs arises from the larger increase in the sharing rate generated by the increase in $p_{H}$. Table 2 illustrates the magnitudes of the relevant effects in the setting where $V=50, I=20$, $\phi_{H}=\phi_{L}=0.5, q=0.65$, and $t_{H}=t_{L}=2.5$. 


\begin{tabular}{|ccccccc|}
\hline$p_{L}$ & $p_{H}$ & $\beta^{*}$ & $\pi^{*}$ & $W_{L}^{*}$ & $W_{H}^{*}$ & $W^{*}$ \\
\hline 0.15 & 0.5 & 0.0763 & 0.3152 & 0.0040 & 0.1536 & 0.4728 \\
0.16 & 0.5 & 0.0755 & 0.3099 & 0.0045 & 0.1505 & 0.4649 \\
0.17 & 0.5 & 0.0748 & 0.3055 & 0.0050 & 0.1478 & 0.4583 \\
0.18 & 0.5 & 0.0742 & 0.3021 & 0.0055 & 0.1456 & 0.4531 \\
0.19 & 0.5 & 0.0738 & 0.2995 & 0.0060 & 0.1437 & 0.4492 \\
0.20 & 0.5 & 0.0734 & 0.2977 & 0.0066 & 0.1423 & 0.4466 \\
\hline
\end{tabular}

Table 2A. The Effects of an Increase in $p_{L}$.

\begin{tabular}{|ccccccc|}
\hline$p_{L}$ & $p_{H}$ & $\beta^{*}$ & $\pi^{*}$ & $W_{L}^{*}$ & $W_{H}^{*}$ & $W^{*}$ \\
\hline 0.15 & 0.50 & 0.0763 & 0.3152 & 0.0040 & 0.1536 & 0.4728 \\
0.15 & 0.51 & 0.0848 & 0.4052 & 0.0050 & 0.1976 & 0.6078 \\
0.15 & 0.52 & 0.0930 & 0.5063 & 0.0060 & 0.2472 & 0.7595 \\
0.15 & 0.53 & 0.1009 & 0.6187 & 0.0070 & 0.3023 & 0.9280 \\
0.15 & 0.54 & 0.1086 & 0.7422 & 0.0081 & 0.3630 & 1.1133 \\
0.15 & 0.55 & 0.1159 & 0.8768 & 0.0093 & 0.4291 & 1.3152 \\
\hline
\end{tabular}

Table 2B. The Effects of an Increase in $p_{H}$.

Table $2 \mathrm{~A}$ reveals that when $p_{H}=0.50$, the welfare of $L$ entrepreneurs $\left(W_{L}^{*}\right)$ increases by $65 \%$, from .0040 to .0066 , as $p_{L}$ increases by five percentage points (or by $33 \%$ ), from .15 to .20. Table $2 \mathrm{~B}$ demonstrates that when $p_{L}=0.15, W_{L}^{*}$ increases by more than $130 \%$, from .0040 to .0093 , as $p_{H}$ increases by five percentage points (or by $10 \%$ ), from .50 to .55 .

\section{Extensions and Conclusions.}

Our simple model of the entrepreneurial sector illustrates that the design of policy to enhance performance in the entrepreneurial sector can entail some subtleties. Our findings indicate, for example, that although it would be natural to expect any policy that enhances 
industry productivity to increase the welfare of some entrepreneurs and/or venture capitalists, this is not necessarily the case. To the contrary, some productivity increases can produce Pareto losses. (Recall Propositions 3 and 4.) Our findings also suggest that if a primary social objective is to increase the welfare of less capable entrepreneurs, this objective may be better pursued in some instances by enhancing the productivity of more capable entrepreneurs than by enhancing the productivity of the less capable entrepreneurs. (Recall Corollary 2.)

For simplicity, the analysis to this point has focused on separate, rather than simultaneous, increases in project success probabilities. In practice, though, a technological change or a general education or training program can enhance the performance of a broad spectrum of entrepreneurial projects. Proposition 5 records one outcome that can arise when $p_{L}$ and $p_{H}$ increase simultaneously in our model.

Proposition 5. Simultaneous increases in the success probabilities of all projects can produce Pareto losses (i.e., $\pi^{*}, W_{L}^{*}$, and $W_{H}^{*}$ can all decline as $p_{L}$ and $p_{H}$ both increase).

Proposition 5 is an immediate corollary of Proposition 4. As long as the increase in $p_{L}$ is sufficiently pronounced relative to the increase in $p_{H}$, the conclusions of Proposition 4 will prevail.

Also for simplicity, we have taken the lender's ability to discern project quality, $q$, to be exogenous. However, many of our key qualitative conclusions hold more generally, as Proposition 6 indicates. The proposition considers the setting with endogenous screening. In this setting, $C(q)$ is the lender's cost of implementing screening accuracy $q . \quad C(\cdot)$ is an increasing, convex function of $q$ that gives rise to a profit function for the lender that is strictly concave in $q$ with an interior optimum $\left(q^{*} \in\left(\frac{1}{2}, 1\right)\right) \cdot{ }^{29}$

\footnotetext{
${ }^{29}$ Sufficient conditions are: (i) $C^{\prime}(q)>0$ and $C^{\prime \prime}(q)>0$ for all $q \in\left(\frac{1}{2}, 1\right)$; (ii) $C\left(\frac{1}{2}\right)=0$; (iii) $\lim _{q \rightarrow \frac{1}{2}}$ $C^{\prime}(q)=0$; and (iv) $\lim _{q \rightarrow 1} C^{\prime}(1)=\infty$.
} 
Proposition 6. The lender's profit $\left(\pi^{*}\right)$ and total welfare $\left(W^{*}\right)$ both decline as $p_{L}$ increases in the setting with endogenous screening when $p_{L}$ is sufficiently small.

Given our focus on changes in entrepreneurial productivity, we have not analyzed the effects of changes in other elements of the model. Before concluding, we mention a somewhat subtle effect of a change in the lender's screening accuracy, which might be viewed as a component of the lender's productivity. Recall that an $L$ entrepreneur is unable to secure funding for his project when the lender assesses the project's quality accurately. Consequently, one might suspect that the welfare of $L$ entrepreneurs $\left(W_{L}^{*}\right)$ will decline as the lender's screening accuracy $(q)$ increases. However, this is not always the case. It can be shown that the lender increases the sharing rate $\left(\beta^{*}\right)$ as $q$ increases. Furthermore, the increase in $\beta^{*}$ can be sufficient to generate an increase in $W_{L}^{*}$, despite the reduced probability that the project of a given $L$ entrepreneur is funded.$^{30}$ This conclusion reinforces the basic message that asymmetric information about likely entrepreneurial performance can introduce important subtleties into an assessment of the welfare implications of improved productivity in the entrepreneurial sector.

Additional extensions of our simple model merit further investigation. For instance, richer payoff structures, more general screening technologies, and expanded project variety might be considered. Risk averse entrepreneurs with varying personal financial resources, multiple lenders, correlation in project qualities, varying and nonlinear investment costs, and entrepreneurial moral hazard might also be analyzed. These extensions of our analysis may introduce additional conclusions of interest. However, the extensions seem unlikely to alter the observation that the impacts of productivity increases in the entrepreneurial sector can be subtle and varied.

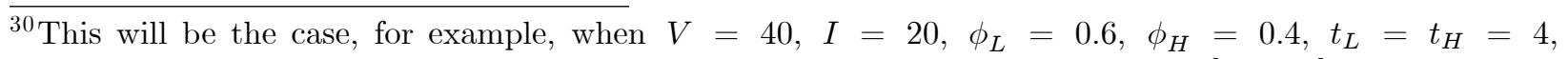
$p_{L}=0.1$, and $p_{H}=0.65$. In this setting, $W_{L}^{*}$ increases as $q$ increases when $q \in[.58, .62]$. 


\section{Appendix}

\section{Proof of Lemma 1.}

Much as in the analysis in the proof of Lemma 2 (below), it is readily verified that the borrower that is indifferent between applying for funding and not applying is located at:

$$
x=\frac{\beta V}{t}\left[\phi_{L} p_{L}(1-q)+\phi_{H} p_{H} q\right] .
$$

Using (10), the lender's (expected) profit when he sets sharing rate $\beta$ is:

$$
\begin{array}{r}
\pi_{S}(\beta)=\frac{\beta V}{t}\left[\phi_{L} p_{L}(1-q)+\phi_{H} p_{H} q\right]\left\{\phi_{L}[1-q]\left[p_{L}(1-\beta) V-I\right]\right. \\
\left.+\phi_{H} q\left[p_{H}(1-\beta) V-I\right]\right\} .
\end{array}
$$

Maximizing $\pi_{S}(\beta)$ with respect to $\beta$ provides:

$$
\beta_{S}=\frac{\phi_{L}[1-q]\left[p_{L} V-I\right]+\phi_{H} q\left[p_{H} V-I\right]}{2 V\left[\phi_{L} p_{L}(1-q)+\phi_{H} p_{H} q\right]}=\frac{1}{2}-\frac{\left[\phi_{L}(1-q)+\phi_{H} q\right] I}{2 V\left[\phi_{L} p_{L}(1-q)+\phi_{H} p_{H} q\right]} .
$$

The conclusions in the Lemma follow immediately from (12).

\section{Proof of Observation 1.}

Substituting (12) into (11) reveals that the lender's (maximum) profit in the symmetric information setting is:

$$
\pi_{S}=\frac{1}{4 t}\left\{\phi_{L}[1-q]\left[p_{L} V-I\right]+\phi_{H} q\left[p_{H} V-I\right]\right\}^{2} .
$$

The welfare of entrepreneurs in this setting is:

$$
\begin{aligned}
W_{E S} & =\left[\phi_{L}(1-q) p_{L}+\phi_{H} q p_{H}\right] \beta V x-\int_{0}^{x} t x d x \\
& =\frac{1}{8 t}\left\{\phi_{L}[1-q]\left[p_{L} V-I\right]+\phi_{H} q\left[p_{H} V-I\right]\right\}^{2} .
\end{aligned}
$$

The equality in (14) follows from (10) and (12). From (13) and (14), welfare in the symmetric information setting is:

$$
W_{S}=\pi_{S}+W_{E S}=\frac{3}{8 t}\left\{\phi_{L}[1-q]\left[p_{L} V-I\right]+\phi_{H} q\left[p_{H} V-I\right]\right\}^{2} .
$$

The conclusions in the Observation follow directly from (15).

\section{Proof of Lemma 2.}

An $L$ entrepreneur's expected payoff from applying for funding is $[1-q] p_{L} \beta V$. The $L$ entrepreneur located farthest from the lender that will apply for funding is the one for whom this expected payoff equals his transactions cost: 


$$
[1-q] p_{L} \beta V=t_{L} x_{L} \Rightarrow x_{L}=\beta p_{L} V\left[\frac{1-q}{t_{L}}\right] .
$$

The analysis for the type $H$ borrower is analogous, and so is omitted.

\section{Proof of Lemma 3.}

Substituting from Lemma 2 into (2) reveals that the lender's profit when he sets sharing rate $\beta$ is:

$$
\pi(\beta)=\left[\frac{\beta V}{t_{L}}\right] \phi_{L} p_{L}[1-q]^{2}\left[p_{L}(1-\beta) V-I\right]+\left[\frac{\beta V}{t_{H}}\right] \phi_{H} p_{H} q^{2}\left[p_{H}(1-\beta) V-I\right] .
$$

Differentiating (16) provides:

$$
\begin{aligned}
\frac{\partial \pi(\cdot)}{\partial \beta}=\frac{V}{t_{L}} \phi_{L} p_{L}[1-q]^{2} & {\left[p_{L} V-I\right]+\frac{V}{t_{H}} \phi_{H} p_{H} q^{2}\left[p_{H} V-I\right] } \\
& -2 \beta V^{2}\left[\phi_{L} p_{L}^{2}(1-q)^{2} \frac{1}{t_{L}}+\phi_{H} p_{H}^{2} q^{2} \frac{1}{t_{H}}\right] .
\end{aligned}
$$

It is readily verified that $\pi(\cdot)$ is a strictly concave function of $\beta$, that $\left.\frac{\partial \pi(\cdot)}{\partial \beta}\right|_{\beta=1}<0$, and that $\left.\frac{\partial \pi(\cdot)}{\partial \beta}\right|_{\beta=0}>0$ when Assumption 1 holds. Therefore, (5) follows directly from (17).

\section{Proof of Lemma 4.}

From (5):

$$
1-\beta^{*}=\frac{V\left[\phi_{L} p_{L}^{2}(1-q)^{2} t_{H}+\phi_{H} p_{H}^{2} q^{2} t_{L}\right]+I\left[\phi_{L} p_{L}(1-q)^{2} t_{H}+\phi_{H} p_{H} q^{2} t_{L}\right]}{2 V\left[\phi_{L} p_{L}^{2}(1-q)^{2} t_{H}+\phi_{H} p_{H}^{2} q^{2} t_{L}\right]} .
$$

From (16):

$$
\begin{aligned}
\pi(\beta)=\frac{\beta V}{t_{L} t_{H}}[1-\beta] V\left[\phi_{L} p_{L}^{2}(1-q)^{2} t_{H}+\phi_{H} p_{H}^{2} q^{2} t_{L}\right] \\
-\frac{\beta V}{t_{L} t_{H}}\left[\phi_{L} p_{L}(1-q)^{2} t_{H}+\phi_{H} p_{H} q^{2} t_{L}\right] I .
\end{aligned}
$$

From (18):

$$
\begin{aligned}
{\left[1-\beta^{*}\right] V\left[\phi_{L} p_{L}^{2}(1-q)^{2} t_{H}+\phi_{H} p_{H}^{2} q^{2} t_{L}\right] } & = \\
& \frac{1}{2} V\left[\phi_{L} p_{L}^{2}(1-q)^{2} t_{H}+\phi_{H} p_{H}^{2} q^{2} t_{L}\right]+\frac{1}{2} I\left[\phi_{L} p_{L}(1-q)^{2} t_{H}+\phi_{H} p_{H} q^{2} t_{L}\right] .
\end{aligned}
$$

From (19) and (20):

$$
\pi\left(\beta^{*}\right)=\frac{\beta^{*} V}{2 t_{L} t_{H}}\left\{V\left[\phi_{L} p_{L}^{2}(1-q)^{2} t_{H}+\phi_{H} p_{H}^{2} q^{2} t_{L}\right]\right.
$$




$$
\left.-I\left[\phi_{L} p_{L}(1-q)^{2} t_{H}+\phi_{H} p_{H} q^{2} t_{L}\right]\right\} .
$$

Relations (5) and (21) imply that $\pi^{*}$ is as specified in (6).

\section{Proof of Lemma 5.}

From (3) and Lemma 2:

$$
\begin{aligned}
W_{L} & =\phi_{L}\left\{[1-q] p_{L} V \beta x_{L}-\int_{0}^{x_{L}} t_{L} x d x\right\}=\phi_{L}\left\{[1-q] p_{L} V \beta x_{L}-\frac{1}{2} t_{L} x_{L}^{2}\right\} \\
& =\phi_{L} \beta p_{L} V\left[\frac{1-q}{t_{L}}\right]\left\{[1-q] p_{L} V \beta-\frac{1}{2} t_{L} \beta p_{L} V\left[\frac{1-q}{t_{L}}\right]\right\} \\
& =\frac{\phi_{L}}{2 t_{L}}[1-q]^{2}\left(\beta p_{L} V\right)^{2} .
\end{aligned}
$$

Substituting from (5) into (22) provides (7). Relation (8) is derived in analogous fashion.

From (6), (7), and (8), equilibrium total welfare is:

$$
\begin{gathered}
W^{*}=W_{L}^{*}+W_{H}^{*}+\pi^{*}=\frac{\left\{\phi_{L} p_{L}[1-q]^{2} t_{H}\left[p_{L} V-I\right]+\phi_{H} p_{H} q^{2} t_{L}\left[p_{H} V-I\right]\right\}^{2}}{8 t_{L} t_{H}\left[\phi_{L} p_{L}^{2}(1-q)^{2} t_{H}+\phi_{H} p_{H}^{2} q^{2} t_{L}\right]^{2}} \\
\cdot\left\{\phi_{L} p_{L}^{2} t_{H}[1-q]^{2}+\phi_{H} p_{H}^{2} q^{2} t_{L}+2\left[\phi_{L} p_{L}^{2} t_{H}[1-q]^{2}+\phi_{H} p_{H}^{2} q^{2} t_{L}\right]\right\} \\
=\frac{3\left\{\phi_{L} p_{L}[1-q]^{2} t_{H}\left[p_{L} V-I\right]+\phi_{H} p_{H} q^{2} t_{L}\left[p_{H} V-I\right]\right\}^{2}}{8 t_{L} t_{H}\left[\phi_{L} p_{L}^{2}(1-q)^{2} t_{H}+\phi_{H} p_{H}^{2} q^{2} t_{L}\right]} \cdot
\end{gathered}
$$

\section{Proof of Lemma 6.}

It is apparent from (5) that $\beta^{*}$ increases as $V$ increases and as $I$ decreases. Furthermore:

$$
\begin{aligned}
\frac{\partial \beta^{*}}{\partial t_{H}} \stackrel{s}{=} & {\left[\phi_{L} p_{L}^{2}(1-q)^{2} t_{H}+\phi_{H} p_{H}^{2} q^{2} t_{L}\right] \phi_{L} p_{L}[1-q]^{2}\left[p_{L} V-I\right] } \\
& -\left\{\phi_{L} p_{L}[1-q]^{2}\left[p_{L} V-I\right] t_{H}+\phi_{H} p_{H} q^{2}\left[p_{H} V-I\right] t_{L}\right\} \phi_{L} p_{L}^{2}[1-q]^{2} \\
= & -\phi_{L} p_{L} \phi_{H} p_{H} q^{2}[1-q]^{2}\left[p_{H}-p_{L}\right] t_{L} I<0 . \\
\frac{\partial \beta^{*}}{\partial t_{L}} \stackrel{s}{=} & {\left[\phi_{L} p_{L}^{2}(1-q)^{2} t_{H}+\phi_{H} p_{H}^{2} q^{2} t_{L}\right] \phi_{H} p_{H} q^{2}\left[p_{H} V-I\right] } \\
& -\left\{\phi_{L} p_{L}[1-q]^{2}\left[p_{L} V-I\right] t_{H}+\phi_{H} p_{H} q^{2}\left[p_{H} V-I\right] t_{L}\right\} \phi_{H} p_{H}^{2} q^{2} \\
= & \phi_{L} p_{L} \phi_{H} p_{H} q^{2}[1-q]^{2}\left[p_{H}-p_{L}\right] t_{H} I>0 .
\end{aligned}
$$




$$
\begin{aligned}
\frac{\partial \beta^{*}}{\partial p_{H}} \stackrel{s}{=} & {\left[\phi_{L} p_{L}^{2}(1-q)^{2} t_{H}+\phi_{H} p_{H}^{2} q^{2} t_{L}\right] \phi_{H} q^{2} t_{L}\left[2 p_{H} V-I\right] } \\
& -2 \phi_{H} p_{H} q^{2} t_{L}\left\{\phi_{L} p_{L}[1-q]^{2}\left[p_{L} V-I\right] t_{H}+\phi_{H} p_{H} q^{2}\left[p_{H} V-I\right] t_{L}\right\} \\
= & I \phi_{H} q^{2} t_{L}\left\{\phi_{L} p_{L}[1-q]^{2} t_{H}\left[2 p_{H}-p_{L}\right]+\phi_{H} p_{H}^{2} q^{2} t_{L}\right\}>0 . \\
\frac{\partial \beta^{*}}{\partial \phi_{H}} \stackrel{s}{=} & {\left[\phi_{L} p_{L}^{2}(1-q)^{2} t_{H}+\phi_{H} p_{H}^{2} q^{2} t_{L}\right]\left\{p_{H} q^{2}\left[p_{H} V-I\right] t_{L}-p_{L}[1-q]^{2}\left[p_{L} V-I\right] t_{H}\right\} } \\
& -\left[p_{H}^{2} q^{2} t_{L}-p_{L}^{2}(1-q)^{2} t_{H}\right]\left\{\phi_{L} p_{L}[1-q]^{2}\left[p_{L} V-I\right] t_{H}+\phi_{H} p_{H} q^{2}\left[p_{H} V-I\right] t_{L}\right\} \\
= & p_{H} q^{2}[1-q]^{2} t_{L} t_{H}\left[p_{H}-p_{L}\right] I>0 .
\end{aligned}
$$

\section{Proof of Lemma 7.}

From (5):

$$
\begin{aligned}
\frac{\partial \beta^{*}}{\partial p_{L}} \stackrel{s}{=} & {\left[\phi_{L} p_{L}^{2}(1-q)^{2} t_{H}+\phi_{H} p_{H}^{2} q^{2} t_{L}\right] \phi_{L}[1-q]^{2} t_{H}\left[2 p_{L} V-I\right] } \\
& -2 \phi_{L} p_{L}[1-q]^{2} t_{H}\left\{\phi_{L} p_{L}[1-q]^{2}\left[p_{L} V-I\right] t_{H}+\phi_{H} p_{H} q^{2}\left[p_{H} V-I\right] t_{L}\right\} \\
= & I \phi_{L}[1-q]^{2} t_{H}\left\{\phi_{L} p_{L}^{2}[1-q]^{2} t_{H}+\phi_{H} p_{H} q^{2} t_{L}\left[2 p_{L}-p_{H}\right]\right\} .
\end{aligned}
$$

From (23):

$$
\begin{aligned}
\frac{\partial \beta^{*}}{\partial p_{L}}>0 & \Leftrightarrow \phi_{L}[1-q]^{2} t_{H} p_{L}^{2}+2 \phi_{H} p_{H} q^{2} t_{L} p_{L}-\phi_{H} p_{H}^{2} q^{2} t_{L}>0 \\
& \Leftrightarrow \frac{\phi_{L}[1-q]^{2} t_{H}}{\phi_{H} q^{2} t_{L}}\left(\frac{p_{L}}{p_{H}}\right)^{2}+2\left(\frac{p_{L}}{p_{H}}\right)-1>0 \\
& \Leftrightarrow \delta_{0} y^{2}+2 y-1>0 \text { where } y=\frac{p_{L}}{p_{H}} \text { and } \delta_{0}=\frac{\phi_{L}[1-q]^{2} t_{H}}{\phi_{H} q^{2} t_{L}} \\
& \Leftrightarrow y>\frac{1}{2 \delta_{0}}\left[-2+\sqrt{4+4 \delta_{0}}\right] \Leftrightarrow y>\delta_{1}=\frac{\sqrt{1+\delta_{0}}-1}{\delta_{0}} .
\end{aligned}
$$

\section{Proof of Proposition 1.}

It is apparent from (6), (7), and (8) that $\frac{\partial \pi^{*}}{\partial V}>0, \frac{\partial W_{L}^{*}}{\partial V}>0, \frac{\partial W_{H}^{*}}{\partial V}>0, \frac{\partial \pi^{*}}{\partial I}<0$, $\frac{\partial W_{L}^{*}}{\partial I}<0$, and $\frac{\partial W_{H}^{*}}{\partial I}<0$. Now define:

$$
z \equiv \phi_{L} p_{L}[1-q]^{2} t_{H}\left[p_{L} V-I\right]+\phi_{H} p_{H} q^{2} t_{L}\left[p_{H} V-I\right]>0 .
$$

The inequality in (24) follows from Assumption 1. Then from (6):

$$
\frac{\partial \pi^{*}}{\partial p_{H}} \stackrel{s}{=}\left[\phi_{L} p_{L}^{2} t_{H}(1-q)^{2}+\phi_{H} p_{H}^{2} t_{L} q^{2}\right] 2 z \phi_{H} q^{2} t_{L}\left[p_{H} V-I\right]-z^{2} 2 \phi_{H} p_{H}^{2} t_{L} q^{2}
$$




$$
\begin{aligned}
& \stackrel{s}{=}\left[\phi_{L} p_{L}^{2} t_{H}(1-q)^{2}+\phi_{H} p_{H}^{2} t_{L} q^{2}\right]\left[p_{H} V-I\right] \\
& \quad-p_{H}\left\{\phi_{L} p_{L} t_{H}[1-q]^{2}\left[p_{L} V-I\right]+\phi_{H} p_{H} t_{L} q^{2}\left[p_{H} V-I\right]\right\} \\
& \quad \phi_{L} p_{L}^{2} t_{H}[1-q]^{2}\left[p_{H} V-I\right]-\phi_{L} p_{L} p_{H} t_{H}[1-q]^{2}\left[p_{L} V-I\right]>0 .
\end{aligned}
$$

The inequality in (25) holds because $p_{H} V-I>0>p_{L} V-I$.

Also from (6):

$$
\begin{aligned}
& \frac{\partial \pi^{*}}{\partial t_{H}} \stackrel{s}{=} z t_{H}\left[\phi_{L} p_{L}^{2} t_{H}(1-q)^{2}+\phi_{H} p_{H}^{2} t_{L} q^{2}\right] 2 \phi_{L} p_{L}[1-q]^{2}\left[p_{L} V-I\right]-z^{2}\left\{2 \phi_{L} p_{L}^{2} t_{H}[1-q]^{2}+\phi_{H} p_{H}^{2} t_{L} q^{2}\right\} \\
& \stackrel{s}{=} 2 \phi_{L} p_{L}[1-q]^{2} t_{H}\left[p_{L} V-I\right]\left[\phi_{L} p_{L}^{2} t_{H}(1-q)^{2}+\phi_{H} p_{H}^{2} t_{L} q^{2}\right] \\
&-\left[2 \phi_{L} p_{L}^{2} t_{H}(1-q)^{2}+\phi_{H} p_{H}^{2} t_{L} q^{2}\right] \\
& \quad \cdot\left\{\phi_{L} p_{L} t_{H}[1-q]^{2}\left[p_{L} V-I\right]+\phi_{H} p_{H} t_{L} q^{2}\left[p_{H} V-I\right]\right\} \\
&=\phi_{L} p_{L}[1-q]^{2} t_{H}\left[p_{L} V-I\right] \phi_{H} p_{H}^{2} t_{L} q^{2} \\
& \quad-\phi_{H} p_{H} t_{L} q^{2}\left[p_{H} V-I\right]\left[2 \phi_{L} p_{L}^{2} t_{H}(1-q)^{2}+\phi_{H} p_{H}^{2} t_{L} q^{2}\right]<0 .
\end{aligned}
$$

From (7):

$$
\begin{aligned}
\frac{\partial W_{L}^{*}}{\partial p_{H}} \stackrel{s}{=} & {\left[\phi_{L} p_{L}^{2}(1-q)^{2} t_{H}+\phi_{H} p_{H}^{2} q^{2} t_{L}\right]^{2} 2 z \phi_{H} q^{2} t_{L}\left[2 p_{H} V-I\right] } \\
& -z^{2} 2\left[\phi_{L} p_{L}^{2}(1-q)^{2} t_{H}+\phi_{H} p_{H}^{2} q^{2} t_{L}\right] 2 \phi_{H} p_{H} q^{2} t_{L} \\
\stackrel{s}{=} & {\left[2 p_{H} V-I\right]\left[\phi_{L} p_{L}^{2}(1-q)^{2} t_{H}+\phi_{H} p_{H}^{2} q^{2} t_{L}\right] } \\
& \quad-2 p_{H}\left\{\phi_{L} p_{L}[1-q]^{2} t_{H}\left[p_{L} V-I\right]+\phi_{H} p_{H} q^{2} t_{L}\left[p_{H} V-I\right]\right\} \\
> & 2\left[p_{H} V-I\right]\left[\phi_{L} p_{L}^{2}(1-q)^{2} t_{H}+\phi_{H} p_{H}^{2} q^{2} t_{L}\right]-2 \phi_{H} p_{H} q^{2} t_{L}\left[p_{H} V-I\right] \\
= & 2\left[p_{H} V-I\right] \phi_{L} p_{L}^{2}[1-q]^{2} t_{H}>0 .
\end{aligned}
$$

The inequality in (27) holds because $2 p_{H} V-I>2\left[p_{H} V-I\right]>0$ and $p_{L} V-I<0$.

Also from (7):

$$
\begin{aligned}
\frac{\partial W_{L}^{*}}{\partial t_{H}} \stackrel{s}{=} & {\left[\phi_{L} p_{L}^{2}(1-q)^{2} t_{H}+\phi_{H} p_{H}^{2} q^{2} t_{L}\right]^{2} 2 z \phi_{L} p_{L}[1-q]^{2}\left[p_{L} V-I\right] } \\
& -z^{2} 2\left[\phi_{L} p_{L}^{2}(1-q)^{2} t_{H}+\phi_{H} p_{H}^{2} q^{2} t_{L}\right] \phi_{L} p_{L}^{2}[1-q]^{2} \\
\stackrel{s}{=} & {\left[\phi_{L} p_{L}^{2}(1-q)^{2} t_{H}+\phi_{H} p_{H}^{2} q^{2} t_{L}\right]\left[p_{L} V-I\right] } \\
& \quad-p_{L}\left\{\phi_{L} p_{L}[1-q]^{2} t_{H}\left[p_{L} V-I\right]+\phi_{H} p_{H} q^{2} t_{L}\left[p_{H} V-I\right]\right\}
\end{aligned}
$$




$$
=\phi_{H} p_{H}^{2} q^{2} t_{L}\left[p_{L} V-I\right]-\phi_{H} p_{H} p_{L} q^{2} t_{L}\left[p_{H} V-I\right]<0 .
$$

The inequality in (28) holds because $p_{H} V-I>0>p_{L} V-I$.

The proof that $\frac{\partial W_{H}^{*}}{\partial p_{H}}>0$ and $\frac{\partial W_{H}^{*}}{\partial t_{H}}>0$ follows from (8) in analogous fashion.

\section{Proof of Proposition 2.}

From (6):

$$
\begin{gathered}
\frac{\partial \pi^{*}}{\partial \phi_{H}} \stackrel{s}{=}\left[\phi_{L} p_{L}^{2} t_{H}(1-q)^{2}+\phi_{H} p_{H}^{2} t_{L} q^{2}\right] 2 z\left\{p_{H} t_{L} q^{2}\left[p_{H} V-I\right]-p_{L} t_{H}[1-q]^{2}\left[p_{L} V-I\right]\right\} \\
\quad-z^{2}\left[p_{H}^{2} t_{L} q^{2}-p_{L}^{2} t_{H}(1-q)^{2}\right] \\
\stackrel{s}{=} 2\left[\phi_{L} p_{L}^{2} t_{H}(1-q)^{2}+\phi_{H} p_{H}^{2} t_{L} q^{2}\right]\left\{p_{H} t_{L} q^{2}\left[p_{H} V-I\right]-p_{L} t_{H}[1-q]^{2}\left[p_{L} V-I\right]\right\} \\
\quad-\left[p_{H}^{2} t_{L} q^{2}-p_{L}^{2} t_{H}(1-q)^{2}\right]\left\{\phi_{L} p_{L} t_{H}[1-q]^{2}\left[p_{L} V-I\right]+\phi_{H} p_{H} t_{L} q^{2}\left[p_{H} V-I\right]\right\} \\
=p_{H} t_{L} q^{2}\left[p_{H} V-I\right]\left\{\left[1+\phi_{L}\right] p_{L}^{2} t_{H}[1-q]^{2}+\phi_{H} p_{H}^{2} t_{L} q^{2}\right\} \\
\quad-p_{L} t_{H}[1-q]^{2}\left[p_{L} V-I\right]\left\{\phi_{L} p_{L}^{2} t_{H}[1-q]^{2}+\left[1+\phi_{H}\right] p_{H}^{2} t_{L} q^{2}\right\}>0 .
\end{gathered}
$$

The inequality in (29) holds because $p_{H} V-I>0>p_{L} V-I$.

From (8):

$$
\begin{aligned}
& \frac{\partial W_{H}^{*}}{\partial \phi_{H}} \stackrel{s}{=}\left[\phi_{L} p_{L}^{2}(1-q)^{2} t_{H}+\phi_{H} p_{H}^{2} q^{2} t_{L}\right]^{2} \\
& \cdot\left\{\phi_{H} 2 z\left[p_{H} q^{2} t_{L}\left(p_{H} V-I\right)-p_{L}(1-q)^{2} t_{H}\left(p_{L} V-I\right)\right]+z^{2}\right\} \\
& -\phi_{H} z^{2} 2\left[\phi_{L} p_{L}^{2}(1-q)^{2} t_{H}+\phi_{H} p_{H}^{2} q^{2} t_{L}\right]\left[p_{H}^{2} q^{2} t_{L}-p_{L}^{2}(1-q)^{2} t_{H}\right] \\
& \stackrel{s}{=}\left[\phi_{L} p_{L}^{2}(1-q)^{2} t_{H}+\phi_{H} p_{H}^{2} q^{2} t_{L}\right]\left[2 \phi_{H} p_{H} q^{2} t_{L}\left(p_{H} V-I\right)-2 \phi_{H} p_{L}(1-q)^{2} t_{H}\left(p_{L} V-I\right)\right] \\
& +z\left\{\phi_{L} p_{L}^{2}[1-q]^{2} t_{H}+\phi_{H} p_{H}^{2} q^{2} t_{L}-2 \phi_{H} p_{H}^{2} q^{2} t_{L}+2 \phi_{H} p_{L}^{2}[1-q]^{2} t_{H}\right\} \\
& =\left[\phi_{L} p_{L}^{2}(1-q)^{2} t_{H}+\phi_{H} p_{H}^{2} q^{2} t_{L}\right]\left[2 \phi_{H} p_{H} q^{2} t_{L}\left(p_{H} V-I\right)-2 \phi_{H} p_{L}(1-q)^{2} t_{H}\left(p_{L} V-I\right)\right] \\
& +\left\{\left[1+\phi_{H}\right] p_{L}^{2}[1-q]^{2} t_{H}-\phi_{H} p_{H}^{2} q^{2} t_{L}\right\} \\
& \cdot\left\{\phi_{L} p_{L}[1-q]^{2} t_{H}\left[p_{L} V-I\right]+\phi_{H} p_{H} q^{2} t_{L}\left[p_{H} V-I\right]\right\} \\
& =\phi_{H} p_{H} q^{2} t_{L}\left[p_{H} V-I\right]\left\{2 p_{L}^{2}[1-q]^{2} t_{H}+\phi_{H} p_{H}^{2} q^{2} t_{L}\right\} \\
& -p_{L}[1-q]^{2} t_{H}\left[p_{L} V-I\right] \phi_{H}\left[2 \phi_{H}+\phi_{L}\right] p_{H}^{2} q^{2} t_{L} \\
& +\phi_{L} p_{L}^{2}[1-q]^{2} t_{H}\left\{\phi_{L} p_{L}[1-q]^{2} t_{H}\left[p_{L} V-I\right]+\phi_{H} p_{H} q^{2} t_{L}\left[p_{H} V-I\right]\right\}>0 .
\end{aligned}
$$

The inequality in (30) holds because $p_{H} V-I>0>p_{L} V-I$ and because $z>0$. 
From (7):

$$
\begin{aligned}
& \frac{\partial W_{L}^{*}}{\partial \phi_{H}} \stackrel{s}{=}\left[\phi_{L} p_{L}^{2}(1-q)^{2} t_{H}+\phi_{H} p_{H}^{2} q^{2} t_{L}\right]^{2} \\
& \cdot\left\{\phi_{L} 2 z\left[p_{H} q^{2} t_{L}\left(p_{H} V-I\right)-p_{L}(1-q)^{2} t_{H}\left(p_{L} V-I\right)\right]-z^{2}\right\} \\
& -\phi_{L} z^{2} 2\left[\phi_{L} p_{L}^{2}(1-q)^{2} t_{H}+\phi_{H} p_{H}^{2} q^{2} t_{L}\right]\left[p_{H}^{2} q^{2} t_{L}-p_{L}^{2}(1-q)^{2} t_{H}\right] \\
& \stackrel{s}{=}\left[\phi_{L} p_{L}^{2}(1-q)^{2} t_{H}+\phi_{H} p_{H}^{2} q^{2} t_{L}\right]\left[2 \phi_{L} p_{H} q^{2} t_{L}\left(p_{H} V-I\right)-2 \phi_{L} p_{L}(1-q)^{2} t_{H}\left(p_{L} V-I\right)\right] \\
& -z\left\{\phi_{L} p_{L}^{2}[1-q]^{2} t_{H}+\phi_{H} p_{H}^{2} q^{2} t_{L}+2 \phi_{L} p_{H}^{2} q^{2} t_{L}-2 \phi_{L} p_{L}^{2}[1-q]^{2} t_{H}\right\} \\
& =\left[\phi_{L} p_{L}^{2}(1-q)^{2} t_{H}+\phi_{H} p_{H}^{2} q^{2} t_{L}\right]\left[2 \phi_{L} p_{H} q^{2} t_{L}\left(p_{H} V-I\right)-2 \phi_{L} p_{L}(1-q)^{2} t_{H}\left(p_{L} V-I\right)\right] \\
& -\left\{\left[\phi_{H}+2 \phi_{L}\right] p_{H}^{2} q^{2} t_{L}-\phi_{L} p_{L}^{2}[1-q]^{2} t_{H}\right\} \\
& \cdot\left\{\phi_{L} p_{L}[1-q]^{2} t_{H}\left[p_{L} V-I\right]+\phi_{H} p_{H} q^{2} t_{L}\left[p_{H} V-I\right]\right\} \\
& =p_{H} q^{2} t_{L}\left[p_{H} V-I\right]\left\{\phi_{L}\left[\phi_{H}+2 \phi_{L}\right] p_{L}^{2}[1-q]^{2} t_{H}-\phi_{H}^{2} p_{H}^{2} q^{2} t_{L}\right\} \\
& -\phi_{L} p_{L}[1-q]^{2} t_{H}\left[p_{L} V-I\right]\left\{\left[3 \phi_{H}+2 \phi_{L}\right] p_{H}^{2} q^{2} t_{L}+\phi_{L} p_{L}^{2}[1-q]^{2} t_{H}\right\} \text {. }
\end{aligned}
$$

Since $p_{H} V-I>0>p_{L} V-I$, (31) implies:

$$
\begin{aligned}
\frac{\partial W_{L}^{*}}{\partial \phi_{H}}>0 \text { if } \phi_{L}\left[\phi_{H}+2 \phi_{L}\right] p_{L}^{2}[1-q]^{2} t_{H}-\phi_{H}^{2} p_{H}^{2} q^{2} t_{L}>0 \\
\Leftrightarrow \frac{\phi_{L}\left[\phi_{H}+2 \phi_{L}\right]}{\phi_{H}^{2}}>\frac{p_{H}^{2} q^{2} t_{L}}{p_{L}^{2}[1-q]^{2} t_{H}} \\
\quad \Leftrightarrow \frac{\left[1-\phi_{H}\right]\left[2-\phi_{H}\right]}{\phi_{H}^{2}}>\frac{p_{H}^{2} q^{2} t_{L}}{p_{L}^{2}[1-q]^{2} t_{H}} .
\end{aligned}
$$

\section{Proof of Proposition 3.}

From (6):

$$
\begin{gathered}
\frac{\partial \pi^{*}}{\partial t_{L}} \stackrel{s}{=} t_{L}\left[\phi_{L} p_{L}^{2} t_{H}(1-q)^{2}+\phi_{H} p_{H}^{2} t_{L} q^{2}\right] z 2 \phi_{H} p_{H} q^{2}\left[p_{H} V-I\right] \\
-z^{2}\left\{t_{L} \phi_{H} p_{H}^{2} q^{2}+\phi_{L} p_{L}^{2} t_{H}(1-q)^{2}+\phi_{H} p_{H}^{2} t_{L} q^{2}\right\} \\
\stackrel{s}{=} 2 \phi_{H} p_{H} q^{2} t_{L}\left[p_{H} V-I\right]\left[\phi_{L} p_{L}^{2} t_{H}(1-q)^{2}+\phi_{H} p_{H}^{2} t_{L} q^{2}\right] \\
-\left\{\phi_{L} p_{L} t_{H}[1-q]^{2}\left[p_{L} V-I\right]+\phi_{H} p_{H} t_{L} q^{2}\left[p_{H} V-I\right]\right\} \\
\cdot\left[\phi_{L} p_{L}^{2} t_{H}(1-q)^{2}+2 \phi_{H} p_{H}^{2} t_{L} q^{2}\right] \\
=\phi_{H} p_{H} q^{2} t_{L}\left[p_{H} V-I\right] \phi_{L} p_{L}^{2} t_{H}[1-q]^{2}
\end{gathered}
$$




$$
-\phi_{L} p_{L} t_{H}[1-q]^{2}\left[p_{L} V-I\right]\left[\phi_{L} p_{L}^{2} t_{H}(1-q)^{2}+2 \phi_{H} p_{H}^{2} t_{L} q^{2}\right]>0 .
$$

From (8):

$$
\begin{aligned}
\frac{\partial W_{H}^{*}}{\partial t_{L}} \stackrel{s}{=}\left[\phi_{L} p_{L}^{2}(1-q)^{2} t_{H}+\phi_{H} p_{H}^{2} q^{2} t_{L}\right]^{2} 2 z \phi_{H} p_{H} q^{2}\left[p_{H} V-I\right] \\
\quad-z^{2} 2\left[\phi_{L} p_{L}^{2}(1-q)^{2} t_{H}+\phi_{H} p_{H}^{2} q^{2} t_{L}\right] \phi_{H} p_{H}^{2} q^{2} \\
\stackrel{s}{=}\left[\phi_{L} p_{L}^{2}(1-q)^{2} t_{H}+\phi_{H} p_{H}^{2} q^{2} t_{L}\right]\left[p_{H} V-I\right] \\
\quad-p_{H}\left\{\phi_{L} p_{L}[1-q]^{2} t_{H}\left[p_{L} V-I\right]+\phi_{H} p_{H} q^{2} t_{L}\left[p_{H} V-I\right]\right\} \\
=\phi_{L} p_{L}^{2}[1-q]^{2} t_{H}\left[p_{H} V-I\right]-\phi_{L} p_{L} p_{H}[1-q]^{2} t_{H}\left[p_{L} V-I\right]>0 .
\end{aligned}
$$

From (9):

$$
\begin{aligned}
\frac{\partial W^{*}}{\partial t_{L}} \stackrel{s}{=} & t_{L}\left[\phi_{L} p_{L}^{2}(1-q)^{2} t_{H}+\phi_{H} p_{H}^{2} q^{2} t_{L}\right] 2 z \phi_{H} p_{H} q^{2}\left[p_{H} V-I\right] \\
& \quad-z^{2}\left[\phi_{L} p_{L}^{2}(1-q)^{2} t_{H}+2 \phi_{H} p_{H}^{2} q^{2} t_{L}\right] \\
\stackrel{s}{=} 2 & \phi_{H} p_{H} q^{2} t_{L}\left[p_{H} V-I\right]\left[\phi_{L} p_{L}^{2}(1-q)^{2} t_{H}+\phi_{H} p_{H}^{2} q^{2} t_{L}\right] \\
& -\left[\phi_{L} p_{L}^{2}(1-q)^{2} t_{H}+2 \phi_{H} p_{H}^{2} q^{2} t_{L}\right] \\
& \cdot\left\{\phi_{L} p_{L}[1-q]^{2} t_{H}\left[p_{L} V-I\right]+\phi_{H} p_{H} q^{2} t_{L}\left[p_{H} V-I\right]\right\} \\
= & \phi_{H} p_{H} q^{2} t_{L}\left[p_{H} V-I\right] \phi_{L} p_{L}[1-q]^{2} t_{H} \\
& -\phi_{L} p_{L}[1-q]^{2} t_{H}\left[p_{L} V-I\right]\left[\phi_{L} p_{L}^{2}(1-q)^{2} t_{H}+2 \phi_{H} p_{H}^{2} q^{2} t_{L}\right]>0 .
\end{aligned}
$$

From (7):

$$
\begin{gathered}
\frac{\partial W_{L}^{*}}{\partial t_{L}} \stackrel{s}{=} t_{L}\left[\phi_{L} p_{L}^{2}(1-q)^{2} t_{H}+\phi_{H} p_{H}^{2} q^{2} t_{L}\right]^{2} 2 z \phi_{H} p_{H} q^{2}\left[p_{H} V-I\right] \\
-z^{2}\left\{2 t_{L}\left[\phi_{L} p_{L}^{2}(1-q)^{2} t_{H}+\phi_{H} p_{H}^{2} q^{2} t_{L}\right] \phi_{H} p_{H}^{2} q^{2}\right. \\
\left.+\left[\phi_{L} p_{L}^{2}(1-q)^{2} t_{H}+\phi_{H} p_{H}^{2} q^{2} t_{L}\right]^{2}\right\} \\
\stackrel{s}{=} 2 \phi_{H} p_{H} q^{2} t_{L}\left[p_{H} V-I\right]\left[\phi_{L} p_{L}^{2}(1-q)^{2} t_{H}+\phi_{H} p_{H}^{2} q^{2} t_{L}\right] \\
-\left[\phi_{L} p_{L}^{2}(1-q)^{2} t_{H}+3 \phi_{H} p_{H}^{2} q^{2} t_{L}\right] \\
\cdot\left\{\phi_{L} p_{L}[1-q]^{2} t_{H}\left[p_{L} V-I\right]+\phi_{H} p_{H} q^{2} t_{L}\left[p_{H} V-I\right]\right\} \\
=\phi_{H} p_{H} q^{2} t_{L}\left[p_{H} V-I\right]\left[\phi_{L} p_{L}^{2}(1-q)^{2} t_{H}-\phi_{H} p_{H}^{2} q^{2} t_{L}\right] \\
-\phi_{L} p_{L}[1-q]^{2} t_{H}\left[p_{L} V-I\right]\left[\phi_{L} p_{L}^{2}(1-q)^{2} t_{H}+3 \phi_{H} p_{H}^{2} q^{2} t_{L}\right] \equiv g\left(\phi_{L}\right) .
\end{gathered}
$$

From (33): 


$$
\begin{aligned}
g\left(\phi_{L}\right) \stackrel{s}{=} p_{H} & q^{2} t_{L}\left[p_{H} V-I\right]\left[r p_{L}^{2}(1-q)^{2} t_{H}-p_{H}^{2} q^{2} t_{L}\right] \\
& -r p_{L}[1-q]^{2} t_{H}\left[p_{L} V-I\right]\left[r p_{L}^{2}(1-q)^{2} t_{H}+3 p_{H}^{2} q^{2} t_{L}\right]
\end{aligned}
$$

where $r=\frac{\phi_{L}}{\phi_{H}}$. Since $p_{L} V-I<0$, it is apparent from (33) and (34) that:

$$
\frac{\partial W_{L}^{*}}{\partial t_{L}}>0 \text { if } r=\frac{\phi_{L}}{\phi_{H}} \geq \frac{p_{H}^{2} q^{2} t_{L}}{p_{L}^{2}[1-q]^{2} t_{H}}
$$

\section{Proof of Proposition 4.}

(i).

$$
\frac{\partial \pi^{*}}{\partial p_{L}}=\frac{N_{1} D_{1}}{2 t_{L}\left[\phi_{L} p_{L}^{2}(1-q)^{2} t_{H}+\phi_{H} p_{H}^{2} q^{2} t_{L}\right]^{2}} \stackrel{s}{=} N_{1} D_{1}
$$

where

$$
N_{1} \equiv \phi_{L}[1-q]^{2}\left\{\phi_{H} p_{H} q^{2} t_{L}\left[p_{H} V-I\right]+\phi_{L} p_{L}[1-q]^{2} t_{H}\left[p_{L} V-I\right]\right\}
$$

and

$$
D_{1} \equiv \phi_{L} p_{L}^{3}[1-q]^{2} t_{H} V+\phi_{H} p_{H} q^{2} t_{L}\left[p_{L} p_{H} V-I\left(p_{H}-p_{L}\right)\right]
$$

Proof. Differentiating (6) provides:

$$
\begin{aligned}
& \frac{\partial \pi^{*}}{\partial p_{L}}=\frac{1}{16 t_{L}^{2} t_{H}^{2}\left[\phi_{L} p_{L}^{2}(1-q)^{2} t_{H}+\phi_{H} p_{H}^{2} q^{2} t_{L}\right]^{2}}\left\{4 t_{L} t_{H}\left[\phi_{L} p_{L}^{2} t_{H}(1-q)^{2}+\phi_{H} p_{H}^{2} t_{L} q^{2}\right]\right. \\
& \cdot 2\left\{V\left[\phi_{L} p_{L}^{2} t_{H}(1-q)^{2}+\phi_{H} p_{H}^{2} t_{L} q^{2}\right]-I\left[\phi_{L} p_{L} t_{H}(1-q)^{2}+\phi_{H} p_{H} t_{L} q^{2}\right]\right\} \\
& \text { · }\left[2 V \phi_{L} p_{L} t_{H}(1-q)^{2}-I \phi_{L} t_{H}(1-q)^{2}\right] \\
& -8 t_{L} t_{H}^{2} \phi_{L} p_{L}[1-q]^{2}\left\{V\left[\phi_{L} p_{L}^{2} t_{H}(1-q)^{2}+\phi_{H} p_{H}^{2} t_{L} q^{2}\right]\right. \\
& \left.\left.-I\left[\phi_{L} p_{L} t_{H}(1-q)^{2}+\phi_{H} p_{H} t_{L} q^{2}\right]\right\}^{2}\right\} \\
& =\frac{V\left[\phi_{L} p_{L}^{2} t_{H}(1-q)^{2}+\phi_{H} p_{H}^{2} t_{L} q^{2}\right]-I\left[\phi_{L} p_{L} t_{H}(1-q)^{2}+\phi_{H} p_{H} t_{L} q^{2}\right]}{2 t_{L} t_{H}\left[\phi_{L} p_{L}^{2}(1-q)^{2} t_{H}+\phi_{H} p_{H}^{2} q^{2} t_{L}\right]^{2}} \\
& \cdot\left\{\left[\phi_{L} p_{L}^{2} t_{H}(1-q)^{2}+\phi_{H} p_{H}^{2} t_{L} q^{2}\right]\left[2 V \phi_{L} p_{L} t_{H}(1-q)^{2}-I \phi_{L} t_{H}(1-q)^{2}\right]\right. \\
& -\phi_{L} p_{L} t_{H}[1-q]^{2}\left\{V\left[\phi_{L} p_{L}^{2} t_{H}(1-q)^{2}+\phi_{H} p_{H}^{2} t_{L} q^{2}\right]\right. \\
& \left.\left.-I\left[\phi_{L} p_{L} t_{H}(1-q)^{2}+\phi_{H} p_{H} t_{L} q^{2}\right]\right\}\right\} \\
& =\frac{N_{1} /\left[\phi_{L}(1-q)^{2}\right]}{2 t_{L} t_{H}\left[\phi_{L} p_{L}^{2}(1-q)^{2} t_{H}+\phi_{H} p_{H}^{2} q^{2} t_{L}\right]^{2}} \\
& \cdot\left\{V \phi_{L}^{2} p_{L}^{3} t_{H}^{2}[1-q]^{4}+V \phi_{L} p_{L} \phi_{H} p_{H}^{2} t_{L} t_{H} q^{2}[1-q]^{2}\right. \\
& \left.-I \phi_{L} \phi_{H} p_{H} t_{L} t_{H} q^{2}[1-q]^{2}\left[p_{H}-p_{L}\right]\right\}
\end{aligned}
$$




$$
=\frac{N_{1} D_{1}}{2 t_{L}\left[\phi_{L} p_{L}^{2}(1-q)^{2} t_{H}+\phi_{H} p_{H}^{2} q^{2} t_{L}\right]^{2}} .
$$

(ii). $\quad D_{1}$ is monotonically increasing in $p_{L}$. Furthermore, there exists a $\widehat{p}_{L} \in\left(0, p_{H}\right)$ such that $D_{1}<0$ for $p_{L} \in\left(0, \widehat{p}_{L}\right)$ and $D_{1}>0$ for $p_{L} \in\left(\widehat{p}_{L}, p_{H}\right)$.

Proof. From (37):

$$
\frac{\partial D_{1}}{\partial p_{L}}=3 \phi_{L} p_{L}^{2}[1-q]^{2} t_{H} V+\phi_{H} p_{H} q^{2} t_{L}\left[p_{H} V+I\right]>0 .
$$

Furthermore, from (37):

$$
\begin{aligned}
& \left.D_{1}\right|_{p_{L}=0}=-\phi_{H} p_{H}^{2} q^{2} t_{L} I<0, \text { and } \\
& \left.D_{1}\right|_{p_{L}=p_{H}}=\phi_{L} p_{H}^{3}[1-q]^{2} t_{H} V+\phi_{H} p_{H}^{3} q^{2} t_{L} V>0 .
\end{aligned}
$$

(iii). There exists a $\widehat{p}_{L} \in\left(0, p_{H}\right)$ such that $\frac{\partial \pi^{*}}{\partial p_{L}}<0$ for $p_{L} \in\left(0, \widehat{p}_{L}\right)$ and $\frac{\partial \pi^{*}}{\partial p_{L}}>0$ for $p_{L} \in\left(\widehat{p}_{L}, p_{H}\right)$.

Proof. The proof follows immediately from (i) and (ii) since $N_{1}>0$ for all $p_{L} \in\left(0, p_{H}\right)$ under Assumption 1.

(iv). From (8):

$$
\begin{aligned}
\frac{\partial W_{H}^{*}}{\partial p_{L}} \stackrel{s}{=} & {\left[\phi_{L} p_{L}^{2}(1-q)^{2} t_{H}+\phi_{H} p_{H}^{2} q^{2} t_{L}\right]^{2} 2 z \phi_{L}[1-q]^{2} t_{H}\left[2 p_{L} V-I\right] } \\
& -z^{2} 2\left[\phi_{L} p_{L}^{2}(1-q)^{2} t_{H}+\phi_{H} p_{H}^{2} q^{2} t_{L}\right] 2 \phi_{L} p_{L}[1-q]^{2} t_{H} \\
\stackrel{s}{=} & {\left[2 p_{L} V-I\right]\left[\phi_{L} p_{L}^{2}(1-q)^{2} t_{H}+\phi_{H} p_{H}^{2} q^{2} t_{L}\right]-2 p_{L} z } \\
= & {\left[2 p_{L} V-I\right]\left[\phi_{L} p_{L}^{2}(1-q)^{2} t_{H}+\phi_{H} p_{H}^{2} q^{2} t_{L}\right] } \\
& -2 p_{L}\left\{\phi_{H} p_{H} q^{2} t_{L}\left[p_{H} V-I\right]+\phi_{L} p_{L}[1-q]^{2} t_{H}\left[p_{L} V-I\right]\right\} \\
= & \phi_{L} p_{L}^{2}[1-q]^{2} t_{H} I-\phi_{H} p_{H} q^{2} t_{L}\left[p_{H}-2 p_{L}\right] I .
\end{aligned}
$$

Relation (39) reveals that $\frac{d W_{H}^{*}}{d p_{L}}<0$ if $p_{L} \leq \frac{I}{2 V}$ (since $z>0$ ). Relation (40) reveals that $\frac{d W_{H}^{*}}{d p_{L}}>0$ if $p_{L}>\frac{1}{2} p_{H}$

(v). From (9):

$$
\begin{aligned}
& \frac{\partial W^{*}}{\partial p_{L}} \stackrel{\stackrel{s}{=}}{ }\left[\phi_{L} p_{L}^{2}(1-q)^{2} t_{H}+\phi_{H} p_{H}^{2} q^{2} t_{L}\right] 2 z \phi_{L}[1-q]^{2} t_{H}\left[2 p_{L} V-I\right] \quad-z^{2} 2 \phi_{L} p_{L}[1-q]^{2} t_{H} \\
& \stackrel{s}{=}\left[2 p_{L} V-I\right]\left[\phi_{L} p_{L}^{2}(1-q)^{2} t_{H}+\phi_{H} p_{H}^{2} q^{2} t_{L}\right]-p_{L} z \\
&=\left[2 p_{L} V-I\right]\left[\phi_{L} p_{L}^{2}(1-q)^{2} t_{H}+\phi_{H} p_{H}^{2} q^{2} t_{L}\right]
\end{aligned}
$$




$$
\begin{aligned}
-p_{L}\left\{\phi_{L} p_{L}[1-q]^{2} t_{H}\left[p_{L} V-I\right]+\phi_{H} p_{H} q^{2} t_{L}\left[p_{H} V-I\right]\right\} \\
=\phi_{H} p_{H} q^{2} t_{L}\left[p_{L} p_{H} V-\left(p_{H}-p_{L}\right) I\right]+\phi_{L} p_{L}^{2}[1-q]^{2} t_{H} p_{L} V
\end{aligned}
$$

Since $z>0,(41)$ implies that $\frac{\partial W^{*}}{\partial p_{L}}<0$ if $p_{L}<\frac{I}{2 V}$. Also, (43) implies that $\frac{\partial W^{*}}{\partial p_{L}}>0$ if $p_{L} p_{H} V>\left[p_{H}-p_{L}\right] I \Leftrightarrow \frac{p_{L} p_{H}}{p_{H}-p_{L}}>\frac{I}{V}$.

(vi). From (7):

$$
\sqrt{W_{L}^{*}}=\left[\frac{[1-q] \sqrt{\phi_{L}}}{\sqrt{8 t_{L}}}\right] \omega\left(p_{L}\right)
$$

where:

$$
\omega\left(p_{L}\right) \equiv \frac{p_{L}\left\{\phi_{L} p_{L}[1-q]^{2} t_{H}\left[p_{L} V-I\right]+\phi_{H} p_{H} q^{2} t_{L}\left[p_{H} V-I\right]\right\}}{\phi_{L} p_{L}^{2}[1-q]^{2} t_{H}+\phi_{H} p_{H}^{2} q^{2} t_{L}} .
$$

From (44) and (45) imply:

$$
\frac{\partial W_{L}^{*}}{\partial p_{L}} \gtreqless 0 \quad \text { as } \frac{\partial \omega\left(p_{L}\right)}{\partial p_{L}} \gtreqless 0
$$

From (45):

$$
\begin{aligned}
\omega\left(p_{L}\right) & =p_{L}\left[V-\left(\frac{\phi_{L} p_{L}[1-q]^{2} t_{H}+\phi_{H} p_{H} q^{2} t_{L}}{\phi_{L} p_{L}^{2}(1-q)^{2} t_{H}+\phi_{H} p_{H}^{2} q^{2} t_{L}}\right) I\right] \\
& =p_{L}\left[V-\left(\frac{\left[\frac{\phi_{L}[1-q]^{2} t_{H}}{\phi_{H} p_{H} q^{2} t_{L}}\right] p_{L}+\left[\frac{\phi_{H} p_{H} q^{2} t_{L}}{\phi_{H} p_{H} q^{2} t_{L}}\right]}{\left[\frac{\phi_{L}(1-q)^{2} t_{H}}{\phi_{H} p_{H} q^{2} t_{L}}\right] p_{L}^{2}+\left[\frac{\phi_{H} p_{H}^{2} q^{2} t_{L}}{\phi_{H} p_{H} q^{2} t_{L}}\right]}\right]\right] .
\end{aligned}
$$

Let $A \equiv \frac{\phi_{L}[1-q]^{2} t_{H}}{\phi_{H} p_{H} q^{2} t_{L}}$. Then, (47) implies:

$$
\begin{aligned}
\omega\left(p_{L}\right) & =p_{L}\left[V-\left(\frac{A p_{L}+1}{A p_{L}^{2}+p_{H}}\right) I\right]=V p_{L}-\left[\frac{A p_{L}^{2}+p_{L}}{A p_{L}^{2}+p_{H}}\right] I \\
& =V p_{L}-\left[\frac{A p_{L}^{2}+p_{H}+p_{L}-p_{H}}{A p_{L}^{2}+p_{H}}\right] I=V p_{L}-\left[1+\frac{p_{L}-p_{H}}{A p_{L}^{2}+p_{H}}\right] I .
\end{aligned}
$$

(48) implies:

$$
\begin{gathered}
\begin{aligned}
\frac{\partial \omega\left(p_{L}\right)}{\partial p_{L}} & =V-\left[\frac{A p_{L}^{2}+p_{H}-\left(p_{L}-p_{H}\right)\left(2 A p_{L}\right)}{\left(A p_{L}^{2}+p_{H}\right)^{2}}\right] I \\
& =\frac{\left[A p_{L}^{2}+p_{H}\right]^{2} V-\left[-A p_{L}^{2}+p_{H}\left(1+2 A p_{L}\right)\right] I}{\left[A p_{L}^{2}+p_{H}\right]^{2}}>0 \\
\text { if } \quad\left[A p_{L}^{2}\right. & \left.+p_{H}\right]^{2} V+\left[A p_{L}^{2}-p_{H}\left(1+2 A p_{L}\right)\right] I \\
& =A^{2} p_{L}^{4} V+A p_{L}^{2} I+p_{H}\left[p_{H} V-I+2 A p_{L}\left(p_{L} V-I\right)\right]>0 .
\end{aligned}
\end{gathered}
$$


Let

$$
\begin{aligned}
h\left(p_{L}\right) & =p_{H} V-I+2 A p_{L}\left[p_{L} V-I\right] \\
& =p_{H} V-I+2 p_{L}\left[p_{L} V-I\right]\left[\frac{\phi_{L}(1-q)^{2} t_{H}}{\phi_{H} p_{H} q^{2} t_{L}}\right] \\
& \stackrel{s}{=} \phi_{H} p_{H} q^{2} t_{L}\left[p_{H} V-I\right]+2 \phi_{L} p_{L}[1-q]^{2} t_{H}\left[p_{L} V-I\right] .
\end{aligned}
$$

Relations (46), (49), (50), and (51) imply that $\frac{\partial W_{L}^{*}}{\partial p_{L}}>0$ when $q$ is sufficiently close to 1 .

(i) - (vi) constitute the proof of all conclusions in the Proposition other than the conclusion about Pareto losses. The Pareto losses identified in the Proposition are illustrated in Table 1 in the text. The data in Table 1 were derived using Mathematica.

\section{Proof of Corollary 1.}

From Assumption 1:

$$
\phi_{H} p_{H}\left[p_{H} V-I\right] t_{L}+\phi_{L} p_{L}\left[p_{L} V-I\right] t_{H}>0 \text {. }
$$

Therefore, $h\left(p_{L}\right)>0$ from (51), and so $\frac{\partial W_{L}^{*}}{\partial p_{L}}>0$ from (46), (49), and (50) if:

$$
q^{2} \geq 2[1-q]^{2} \Leftrightarrow q \geq \frac{\sqrt{2}}{1+\sqrt{2}}=0.58579
$$

\section{Proof of Corollary 2.}

The data in Table 2 provide a proof of the corollary.

\section{Proof of Proposition 5.}

As noted in the text, the proposition is an immediate corollary of Proposition 4 since the relevant increase in $p_{L}$ can be made arbitrarily large relative to the increase in $p_{H}$.

\section{Proof of Proposition 6.}

From (6), the lender's profit in this setting when she implements screening accuracy $q$ is:

$$
\Pi(q, C)=\pi^{*}(q)-C(q),
$$

where:

$$
\pi^{*}(q)=\frac{\left[\phi_{H} p_{H} q^{2} t_{L}\left(p_{H} V-I\right)+\phi_{L} p_{L}(1-q)^{2} t_{H}\left(p_{L} V-I\right)\right]^{2}}{4 t_{L} t_{H}\left[\phi_{H} p_{H}^{2} q^{2} t_{L}+\phi_{L} p_{L}^{2}(1-q)^{2} t_{H}\right]} .
$$

Let $q^{*}=\underset{q}{\arg \max } \Pi(q, C)$. Also let $\Pi^{*}=\Pi\left(q^{*}, C\right)$. Then:

$$
\frac{d \Pi^{*}}{d p_{L}}=\left.\frac{\partial \Pi(\cdot)}{\partial p_{L}}\right|_{q=q^{*}}+\left\{\left.\frac{\partial \Pi(\cdot)}{\partial q}\right|_{q=q^{*}}\right\} \frac{\partial q^{*}}{\partial p_{L}}=\left.\frac{\partial \Pi(\cdot)}{\partial p_{L}}\right|_{q=q^{*}} .
$$


The last equality in (54) reflects the envelope theorem. Relations (52) and (54) imply that $\frac{d \Pi^{*}}{d p_{L}} \stackrel{s}{=} \frac{\partial \pi^{*}}{\partial p_{L}}$.

From (9), total welfare in this setting when the lender implements screening accuracy $q$ at cost $C(q)$ is:

$$
\widehat{W}(q, C)=\frac{3\left[\phi_{H} p_{H} q^{2} t_{L}\left(p_{H} V-I\right)+\phi_{L} p_{L}(1-q)^{2} t_{H}\left(p_{L} V-I\right)\right]^{2}}{8 t_{H} t_{L}\left[\phi_{H} p_{H}^{2} q^{2} t_{L}+\phi_{L} p_{L}^{2}(1-q)^{2} t_{H}\right]}-C(q) .
$$

From (52), (53), and (55):

$$
\begin{aligned}
\widehat{W}(q, C) & =\frac{3}{2} \pi^{*}(q)-C(q)=\frac{3}{2}\left[\pi^{*}(q)-\frac{2}{3} C(q)\right] \\
& =\frac{3}{2}\left[\pi^{*}(q)-\widetilde{C}(q)\right]=\frac{3}{2} \Pi(q, \widetilde{C}), \quad \text { where } \widetilde{C}(q)=\frac{2}{3} C(q) .
\end{aligned}
$$

Relation (56) implies:

$$
\begin{aligned}
{\left[\frac{2}{3}\right] \frac{d \widehat{W}(q, C)}{d p_{L}} } & =\frac{d \Pi(q, \widetilde{C})}{d p_{L}}=\left.\frac{\partial \Pi(q, \widetilde{C})}{\partial p_{L}}\right|_{q=q^{*}}=\left.\frac{\partial \pi^{*}(q)}{\partial p_{L}}\right|_{q=q^{*}} \\
& =\left.\frac{2}{3} \frac{\partial \widehat{W}(q, C)}{\partial p_{L}}\right|_{q=q^{*}}=\frac{2}{3} \frac{\partial W^{*}}{\partial p_{L}}
\end{aligned}
$$

The second equality in (57) reflects the envelope theorem. (57) implies that $\frac{d \widehat{W}(q, C)}{d p_{L}} \stackrel{s}{=} \frac{\partial W^{*}}{\partial p_{L}}$. Therefore, the conclusion in the proposition follows from Proposition 4. 


\section{References}

Abramovsky, Laura, Erich Battistin, Emla Fitzsimons, Alissa Goodman, and Helen Simpson, "Providing Employers with Incentives to Train Low-Skilled Workers: Evidence from the UK Employer Training Pilots," Journal of Labor Economics, 29(1), January 2011, 153-193.

Anant, T., Kaushik Basu, and Badal Mukherji, "A Model of Monopoly with Strategic Government Intervention," Journal of Public Economics, 57(1), May 1995, 25-43.

Bose, Arup, Debashis Pal, and David Sappington, "Pareto-Improving Inefficiency," Oxford Economic Papers, 2011 (forthcoming, doi: 10.1093/oep/gpq009).

Fairlie, Robert, The Kauffman Index of Entrepreneurial Activity, 1996-2009, May 2010 (available at http://www.kauffman.org/uploadedfiles/kiea_2010_report.pdf).

Fletcher, Deborah and Steven Slutsky, "Bad Government Can be Good Politics: Political Reputation, Negative Campaigning, and Strategic Shirking," B.E. Journal of Theoretical Economics, 10(1), January 2010, Article 22 (Contributions).

Gelman, Judith and Steven Salop, "Judo Economics: Capacity Limitation And Coupon Competition," Bell Journal of Economics, 14(2), Autumn 1983, 315-325.

Gupta, Barnali, Amoz Katz, and Debashis Pal, "Upstream Monopoly, Downstream Competition and Spatial Price Discrimination," Regional Science and Urban Economics, 24(5), October 1994, 529-542.

Gupta, Barnali, John Heywood, and Debashis Pal, "Strategic Behavior Downstream and the Incentive to Integrate: A Spatial Model with Delivered Pricing," International Journal of Industrial Organization, 13(3), September 1995, 327-334.

Heckman, James, "What Should Be our Human Capital Investment Policy?" Fiscal Studies, 19(2), May 1998, 103-19.

Heckman, James, Robert LaLonde, and Jeffrey Smith, "The Economics and Econometrics of Active Labor Market Programs," in Handbook of Labor Economics, Vol. 3, edited by Orley Ashenfelter and David Card. Amsterdam: Elsevier Science, 1999.

Joskow, Paul and Edward Kahn, "A Quantitative Analysis of Pricing Behavior in California's Wholesale Electricity Market During Summer 2000," Energy Journal, 23(4), October 2002, $1-35$.

Krueger, Alan, and Cecilia Rouse, "The Effect of Workplace Education on Earnings, Turnover, and Job Performance," Journal of Labor Economics, 16(1), January 1998, 61-94.

Leuven, Edwin, and Hessel Oosterbeek, "Evaluating the Effect of Tax Deductions on Training," Journal of Labor Economics, 22(2), April 2004, 461-88.

Lipsey, R. G. and Kelvin Lancaster, "The General Theory of Second Best," Review of Economic Studies, 24(1), 1956-7, 11-32. 